\title{
Hedging under an expected loss constraint with small transaction costs *
}

\author{
Bruno Bouchard $^{\dagger} \quad$ Ludovic Moreau ${ }^{\ddagger} \quad$ H.Mete Soner ${ }^{\S}$
}

May 9, 2018

\begin{abstract}
We consider the problem of option hedging in a market with proportional transaction costs. Since super-replication is very costly in such markets, we replace perfect hedging with an expected loss constraint. Asymptotic analysis for small transaction costs is used to obtain a tractable model. A general expansion theory is developed using the dynamic programming approach. Explicit formulae are obtained in the special cases of exponential and power utility functions. As a corollary, we retrieve the asymptotics for the exponential utility indifference price.
\end{abstract}

Keywords: Expected loss constraint, hedging, transaction cost, asymptotic expansion.

AMS 2000 Subject Classification 60G42; 91B28; 93E20; 49L20

\section{Introduction}

As well known, in a complete market with no frictions, every contingent claim can be replicated by continuous trading of the underlying asset. These replicating strategies however typically yield portfolio processes that are of unbounded variations. Hence, any size of transaction cost renders this portfolio to have an infinite trading cost. Indeed, it has been shown that, generically, the cheapest super-replicating portolio is the simple buy and hold strategy leading to a prohibitive cost [50, 42, 15, 19, 23, 37, 40, 41.

Theoretically almost sure replication is an appealing concept which has been extensively studied in the literature. Firstly, it provides the initial building block for the utility maximization problems by providing the exact description of the wealth processes that enter into the maximization. Also it provides complete risk aversion agreeing with all other approaches and in incomplete markets it yields the pricing intervals. When this interval is tight, it can also have practical uses. However, since this is not the case in markets with transaction costs, one has to consider instead expected loss criteria related to the risk attitude of the investors.

In the frictionless Black-Scholes market Föllmer and Leukert [27, 28] studied the quantile and expected shortfall by exploiting the deep connection to the NeymanPearson lemma, which applies to general complete markets. A more general approach

* Research of the first author is partly supported by ANR Liquirisk and Labex ECODEC. Research of the last two authors was partly supported by the European Research Council under the grant 228053-FiRM, by the ETH Foundation and by the Swiss Finance Institute.

${ }_{\dagger}$ CEREMADE, Université Paris Dauphine and CREST-ENSAE, bouchard@ceremade.dauphine.fr.

${ }_{\ddagger}$ Department of Mathematics, ETH Zurich , ludovic.moreau@math.ethz.ch.

$\S$ Department of Mathematics, ETH Zurich \& Swiss Finance Institute, hmsoner@ethz.ch 
for Markovian settings was then developed in [12, 9, 44, 13 , for diverse markets including jumps and several loss criteria. A particular application of this approach is the utility indifference as introduced by Hodges and Neuberger 34 in which the hedging constraint is given through the maximum utility that one may achieve without the liability. However, in the general formulation of hedging with expected loss, one can place more than one constraint [16] and consider markets with general dynamics as well as frictions.

In this paper, we follow the problem formulation of [12] and develop a coherent asymptotic theory for hedging problems under an expected loss criterion, when the transaction cost is small. Asymptotic analysis allows for more tractable formulae. Our methodology is robust enough to treat models with general dynamics and many loss criteria. For modeling the financial market, we follow the seminal papers [43, 17] and the rigorous mathematical approaches of [22, 24, 49]. For further information on utility maximization under transaction costs, we refer the reader to the book [38] and the references therein.

On the technical side, we build upon the similar theory that was developed in the case of the classical utility maximization. For this problem, an extensive theory is now available starting with the appendix of 49 . There are now many rigorous results [1, 3, 6, 7, 32, 35, 45, 48, 53, as well as interesting formal derivations [2, 33, 54]. The partial differential equation (PDE) technique that we use has its origins in a recent paper [53]. It is based on the theory of the viscosity approach to homogenization of Evans [26. This methodology allows for a flexible asymptotic theory that applies to markets with multiple assets [48, fixed transaction costs [1] and market impact in factor models [45]. A related asymptotic analysis is carried out for stochastic volatility models with different time scales 30, 31, and for utility maximization asymptotics 29. They also use viscosity solution tools, but their methodology is different.

The asymptotic expansion is derived directly using the PDE characterization of the expected loss based price. This equation follows from the stochastic target formulation with controlled expected loss as in 12. In the frictionless case, the problem described in subsection 2.2 is

$$
\pi(t, s, p):=\inf \left\{z \in \mathbb{R}: \mathbb{E}\left[\Psi\left(Z_{T}^{t, s, z, \vartheta}-g\left(S_{T}^{t, s}\right)\right)\right] \geq p \text { for some } \vartheta \in \mathcal{U}(t, s, z)\right\},
$$

where $\Psi$ is the given expected loss function, $p$ is the given desired threshold, $g$ is option pay-off, $\mathcal{U}(t, s, z)$ is the set of admissible controls and the process $Z^{t, s, z, \vartheta}$ is the value of the portfolio with initial stock value $s$, initial wealth value $z$ and control process $\vartheta$. The diffusion type dynamics of $Z^{t, s, z, \vartheta}$ and the exact description of the admissible class $\mathcal{U}(t, s, z)$ are given in section 2 below. Then, with the help of the martingale representation, [12] converts this problem into a standard stochastic target problem introduced in [51, 52. The model with transaction costs is introduced in Section 2.1 and the corresponding dynamic programming equation is a quasi-variational inequality (2.7).

The main result of the paper, outlined in Section 3 is the asymptotic expansion (3.1). It is proved under the hypothesis of Theorem 3.7 and states that the loss due to frictions is proportional to the $2 / 3$ power of the proportional transaction cost and the coefficient of the first term in the expansion is characterized. Although our result is proved for a single risk criteria, it can be generalized to the multi-criteria case by exactly following the steps of [16. This extension naturally increases the dimension of the corresponding PDE but does not introduce any additional technical difficulties.

In the case of exponential and power utility functions, $\Psi$, explicit formulae are available. We collect them in Section 4 In Section 7, we also explain how to construct almost optimal strategies. 
In particular, if one chooses the threshold $p$ to be the value function of the same utility maximization problem with transaction costs but without any liability, one recovers the utility indifference price and its asymptotics. In this context this price was first studied by 21. In the case of an exponential utility, they obtained the price as the difference of two functions. These functions are related to the maximum utility of two similar problems whose solutions are described through a nonlinear parabolic equation with gradient constraints. Related asymptotic formulae were formally derived in [54] and only recently were proved rigorously by Bichuch in [5. Later [46] used an approach similar to ours for this problem. As discussed above, the problem we study is equivalent to hedging the option not perfectly but with a prescribed expected loss. As a consequence, our results described in Section 4 yield the asymptotic formula of [5].

The paper is organized as follows. The next section describes the model and its frictionless counterpart. In Section 3, we state the main theorem and our assumptions. We illustrate this result in the cases of exponential and power utilities in Section 4 Section 5 is devoted to the proof of the main theorem and Section 7 verifies the assumptions in the examples. In Section [6] we prove several technical estimates.

Notations: Given $\mathcal{O} \subset \mathbb{R}^{k}$ and a smooth function $\varphi:\left(t, x^{1}, \ldots, x^{k}\right) \in[0, T] \times \mathcal{O} \mapsto \mathbb{R}$, we write $\varphi_{t}$ and $\varphi_{x^{i}}$ for the partial derivatives with respect to $t$ and $x^{i}$. Second order derivatives are denoted by $\varphi_{x^{i} x^{j}}$, and so on... We use the notations $D \varphi$ and $D^{2} \varphi$ to denote the gradient and the Hessian matrix with respect to the space component $\left(x^{1}, \ldots, x^{k}\right)$. If we want to define them with respect to a subfamily, say $\left(x^{1}, \cdots, x^{i}\right)$, we write $D_{\left(x^{1}, \cdots, x^{i}\right)} \varphi$ and $D_{\left(x^{1}, \cdots, x^{i}\right)}^{2} \varphi$. When $\varphi$ depends on only one variable, we simply write $\varphi^{\prime}$ and $\varphi^{\prime \prime}$ for the first and second order derivatives. Any element of $\mathbb{R}^{k}$ is viewed as a column vector, and ${ }^{\top}$ denotes the transposition. For an element $\zeta \in \mathbb{R}^{k}$ and $r>0$, the open ball of radius $r>0$ centered at $\zeta$ is denoted by $B_{r}(\zeta)$. We let $\bar{B}$ and $\operatorname{Int}(B)$ denote the closure and the interior of $B$. Assertions involving random variables have to be understood in the a.s. sense, if nothing else is specified.

\section{Partial hedging under expected loss constraints and pricing equations}

As usual, we let $(\Omega, \mathcal{F}, \mathbb{P})$ be a complete probability space supporting a one dimensional Brownian motion $W, \mathbb{F}:=\left(\mathcal{F}_{t}\right)_{t \leq T}$ be the right-continuous augmented filtration generated by $W$ and $T>0$ be the fixed time horizon.

\subsection{Controlled loss pricing with proportional transaction costs}

We consider a financial market which consists of a single risky asset $S$, called stock hereafter. For ease of notations, we assume that the risk free interest rate is 0 . Given initial data $(t, s) \in[0, T] \times(0, \infty)$, we let $S^{t, s}$ describe the evolution of this asset, and we assume that it follows the dynamics

$$
S^{t, s}=s+\int_{t}^{\cdot} S_{\tau}^{t, s} \mu\left(\tau, S_{\tau}^{t, s}\right) d \tau+\int_{t}^{\cdot} S_{\tau}^{t, s} \sigma\left(\tau, S_{\tau}^{t, s}\right) d W_{\tau}
$$

in which

$$
\begin{gathered}
(t, s) \in[0, T] \times(0, \infty) \mapsto(s \mu(t, s), s \sigma(t, s)) \in \mathbb{R} \times(0, \infty) \\
\text { is Lipschitz continuous in } s \text { and continuous in } t
\end{gathered}
$$

The latter condition implies the existence and uniqueness of a strong solution. 
Transactions on this market are subject to a proportional cost 11 described by a parameter $\epsilon^{3}>0$. We use the notation $\epsilon$ because we will be interested by the asymptotic $\epsilon \rightarrow 0$. The scaling $\epsilon^{3}$ is just for notational convenience, as it will be clear later on.

As usual in the presence of transaction costs, a portfolio process has to be described by a two dimensional process $(Y, X)$ in which $Y$ denotes the cash account and $X$ denotes the amount of money invested in the stock. We therefore call $(y, x) \in \mathbb{R}^{2}$ an initial endowment at time $t$ if $y$ is the position in cash and $x$ is the amount invested in the stock at time $t$. Then, a financial strategy is an adapted process $L$ with bounded variations. The quantity $L_{\tau}-L_{t-}$ must be interpreted as the cumulated amount of money transferred on the time interval $[t, \tau]$ from the cash account into the account invested in the stock. It admits the canonical decomposition into two non-decreasing adapted processes $L=L^{+}-L^{-}$. We denote by $\mathfrak{L}$ the collection of trading strategies.

Given an initial endowment $(y, x)$ at time $t$, the portfolio process $\left(Y^{t, y, \epsilon, L}, X^{t, x, s, L}\right)$ associated to the strategy $L \in \mathfrak{L}$ evolves according to

$$
\begin{gathered}
Y^{t, y, \epsilon, L}=y-\int_{t}^{\cdot}\left(1+\epsilon^{3}\right) d L_{\tau}^{+}+\int_{t}^{\cdot}\left(1-\epsilon^{3}\right) d L_{\tau}^{-}, \\
X^{t, x, s, L}=x+\int_{t}^{\cdot} X_{\tau}^{t, x, s, L} \frac{d S_{\tau}^{t, s}}{S_{\tau}^{t, s}}+\int_{t}^{\cdot} d L_{\tau}^{+}-\int_{t} d L_{\tau}^{-} .
\end{gathered}
$$

In order to rule out any possible arbitrage, we restrict the set of admissible strategies to the elements of $\mathfrak{L}$ such that the liquidation value of the portfolio is bounded from below, i.e. $L \in \mathcal{L}$ is admissible if there exists $c_{L} \geq 0$ such that

$$
Y^{t, y, \epsilon, L}+\ell^{\epsilon}\left(X^{t, x, s, L}\right) \geq-c_{L} \text { on }[t, T],
$$

where

$$
\ell^{\epsilon}: r \in \mathbb{R} \mapsto r-\epsilon^{3}|r|
$$

We denote by $\mathfrak{L}^{\epsilon}(t, s, y, x)$ the set of admissible strategies associated to the initial data $(s, y, x)$ at time $t$.

We now consider a trader whose aim is to hedge a plain vanilla European option with payoff function $g: r \in(0, \infty) \mapsto g(r) \in \mathbb{R}$. Hereafter, $g$ is assumed to be continuous with linear growth. In general, super-hedging in the presence of proportional transaction costs is much too expensive to make sense in practice, see [20, 42, 50, and [15] for the multivariate setting. We therefore introduce a risk criteria under which the pricing and the hedging of the option will be performed. It is specified through a map $\Psi: r \in$ $\mathbb{R} \mapsto \Psi(r) \in(-\infty, 0]$, which we call loss function. We assume that $\Psi$ is concave2, nondecreasing, continuous on its domain, that $\operatorname{Im}(\Psi):=\{\Psi(r), r \in \mathbb{R}$ s.t. $\Psi(r)>-\infty\}$ is open and that

$$
\mathbb{E}\left[\Psi\left(-g\left(S_{T}^{t, s}\right)\right)\right]>-\infty \quad \text { for all }(t, s) \in[0, T] \times(0, \infty) .
$$

The hedging price associated to the loss function $\Psi$ and a threshold $p \in \operatorname{Im}(\Psi)$ is then defined by

$$
v^{\epsilon}(t, s, p, x):=\inf \left\{y \in \mathbb{R}: \exists L \in \mathfrak{L}^{\epsilon}(t, s, y, x) \text { s.t. } \mathbb{E}\left[\Psi\left(\Delta_{t, s, y, x}^{\epsilon, L}\right)\right] \geq p\right\},
$$

where

$$
\Delta_{t, s, y, x}^{\epsilon, L}:=Y_{T}^{t, y, \epsilon, L}+\ell^{\epsilon}\left(X_{T}^{t, x, s, L}\right)-g\left(S_{T}^{t, s}\right) .
$$

\footnotetext{
${ }^{1}$ See 38 for a general presentation of models with proportional transaction costs.

${ }^{2}$ We make this assumption to obtain the representation in Proposition 2.2 This representation is then used to verify the assumptions. Hence, the main result applies to general loss functions provided the assumptions are verified.
} 
The value $v^{\epsilon}(t, s, p, x)$ is the minimal initial price at which the option with payoff $g\left(S_{T}^{t, s}\right)$ should be sold in order to ensure that the expected loss, as evaluated through $\Psi$, is not below the threshold $p$. Note that the assumption that $\Psi$ is bounded from above is rather natural since we consider here a risk criterion, i.e. one should not have the possibility of compensating losses by unbounded gains. From the mathematical point, it could be relaxed up to additional integrability conditions ensuring that the corresponding optimization problem Max $\mathbb{E}\left[\Psi\left(\Delta_{t, s, y, x}^{\epsilon, L}\right)\right]$ over $L \in \mathfrak{L}^{\epsilon}(t, s, y, x)$ is well-posed, see e.g. [8] and the references therein. Also note that this problem is of interest even in the degenerate case $g \equiv 0$. Then, $v^{\epsilon}$ represents the threshold under which the cash account should not go in order for the terminal wealth to satisfy the requirement in (2.4). This threshold is a building block for the analysis of optimal investment problems under risk constraints, see [10, 14].

The problem (2.4) is a stochastic target problem with controlled loss in the terminology of [12]. In order to obtain a pde characterization, the first step of their analysis consists of increasing the dimension of the state space and of the set of controls in order to turn the target problem under controlled loss in (2.4) into a target problem with $\mathbb{P}$-a.s. terminal constraint in the form of [51, 52]. Namely, $v^{\epsilon}$ admits the equivalent formulation

$v^{\epsilon}(t, s, p, x)=\inf \left\{y \in \mathbb{R}: \exists(L, \alpha) \in \mathfrak{L}^{\epsilon}(t, s, y, x) \times \mathfrak{A}\right.$ s.t. $\left.\Psi\left(\Delta_{t, s, y, x}^{\epsilon, L}\right) \geq P_{T}^{t, p, \alpha}\right\}$

where $\mathfrak{A}$ denotes the set of a.s. square integrable predictable processes such that

$$
P^{t, p, \alpha}:=p+\int_{t}^{\cdot} \alpha_{\tau} d W_{\tau} \quad \text { is a martingale on }[t, T] .
$$

One direction follows by taking expectation, the other one is just a consequence of the martingale representation theorem applied to $\Psi\left(\Delta_{t, s, y, x}^{\epsilon, L}\right)$. Since $\operatorname{Im}(\Psi)$ is convex, by the continuity of $\Psi$ on its domain, it is not difficult to see that we can even restrict the martingale $P^{t, p, \alpha}$ to take values in $\operatorname{Im}(\Psi)$, see [12, 44].

Note that this reformulation is natural. Indeed, the expectation in (2.4) has to be understood as a conditional expectation given the (trivial) information at the starting point $t$. The conditional expectation evolves as time passes, and has no reason to stay above the initial threshold $p$. The martingale process $P^{t, p, \alpha}$ is here to take this evolution into account and turns the problem into a time-consistent one: it describes the evolution of the conditional expectation of $\Psi\left(\Delta_{t, s, y, x}^{\epsilon, L}\right)$.

A geometric dynamic programming principle for problems of the form (2.5) was first obtained by [51, 52. In the present framework, in which controls are of bounded variation, it was further studied by [9]. Up to slight modifications, see the Appendix, it follows from the analysis in $\left[9\right.$ ] that $v^{\epsilon}$ is a (discontinuous) viscosity solution on $\mathfrak{D} \times \mathbb{R}$ of

$$
\begin{gathered}
\max \left\{-\mathcal{L}_{\mathrm{Sx}} \varphi-\hat{\mathcal{L}}_{\mathrm{P} \mid \mathrm{Sx}} \varphi,-\epsilon^{3}+1+\varphi_{x},-\epsilon^{3}-\left(1+\varphi_{x}\right)\right\}=0 \text { on } \mathfrak{D}_{<T} \times \mathbb{R}, \\
\Psi\left(\varphi+x-\epsilon^{3}|x|-g\right)=p \text { on } \mathfrak{D}_{T} \times \mathbb{R}
\end{gathered}
$$

in which we use the notations

$\mathfrak{D}_{<T}:=[0, T) \times(0, \infty) \times \operatorname{Im}(\Psi), \mathfrak{D}_{T}:=\{T\} \times(0, \infty) \times \operatorname{Im}(\Psi), \quad \mathfrak{D}:=\mathfrak{D}_{<T} \cup \mathfrak{D}_{T}$,

and

$$
\begin{gathered}
\mathcal{L}_{\mathrm{P} \mid \mathrm{SX}}^{a} \varphi:=\frac{1}{2} a\left(\bar{\sigma}_{a}+\bar{\sigma}_{0}\right)^{\top} D \varphi_{p} \\
\hat{\mathcal{L}}_{\mathrm{P} \mid \mathrm{SX}} \varphi:=\inf \left\{\mathcal{L}_{\mathrm{P} \mid \mathrm{SX}}^{a} \varphi: a \in \mathbb{R} \text { s.t. } \bar{\sigma}_{a}^{\top} D \varphi=0\right\} \\
\mathcal{L}_{\mathrm{SX}} \varphi:=\varphi_{t}+\bar{\mu}^{\top} D \varphi+\frac{1}{2} \operatorname{Tr}\left[\bar{\sigma}_{0} \bar{\sigma}_{0}^{\top} D^{2} \varphi\right]
\end{gathered}
$$


where $D \varphi_{p}$ is vector of the derivatives of the partial derivative $\varphi_{p}$ and for a given point $(t, s, x, a) \in[0, T] \times(0, \infty) \times \mathbb{R} \times \mathbb{R}$,

$$
\bar{\mu}(t, s, x):=\left(\begin{array}{c}
s \mu(t, s) \\
x \mu(t, s) \\
0
\end{array}\right) \quad \text { and } \quad \bar{\sigma}_{a}(t, s, x):=\left(\begin{array}{c}
s \sigma(t, s) \\
x \sigma(t, s) \\
a
\end{array}\right) .
$$

Theorem 2.1. Assume that $v^{\epsilon}$ is locally bounded. Then, it is a discontinuous viscosity solution of (2.7).

The above characterization can be exploited to compute the pricing function $v^{\epsilon}$ numerically. However, it should be observed that the operator $\hat{\mathcal{L}}_{\mathrm{P} \mid \mathrm{Sx}}$ involves an optimization over the unbounded set $\mathbb{R}$, which makes it discontinuous, and possibly difficult to handle numerically. Moreover, except if $v^{\epsilon}$ is smooth, the above pde does not allow to recover the associated hedging strategy.

In this paper, we follow the approach of [53, and try to provide an expansion of $v^{\epsilon}$ around $\epsilon=0$, i.e. for small values of the transaction costs. For $\epsilon=0$, the financial market is complete and the problem can be solved explicitly by tools from convex analysis as described in the next subsection. We can therefore hope to obtain an explicit expansion, or at least a characterization of the different terms in the expansion which will be more tractable from the numerical point of view.

\subsection{The frictionless benchmark case}

We now consider the frictionless case which will be used to provide an expansion of $v^{\epsilon}$. We refer to [27, 28, for a general exposition of quantile and loss hedging problems in this context, see also [11].

Let $\mathcal{U}$ denote the set of $\mathbb{R}$-valued progressively-measurable and a.s. square integrable processes. Elements of $\mathcal{U}$ will be interpreted as amounts of money invested in the risky asset $S$. Given an initial allocation in amount of cash $z$ at time $t$ and $\vartheta \in \mathcal{U}$, the corresponding (frictionless) wealth process $Z^{t, s, z, \vartheta}$ evolves according to

$$
Z^{t, s, z, \vartheta}=z+\int_{t} \vartheta_{\tau} d S_{\tau}^{t, s} / S_{\tau}^{t, s},
$$

and the analog of $v^{\epsilon}(t, s, p, 0)$ in (2.4) is

$$
\pi(t, s, p):=\inf \left\{z \in \mathbb{R}: \mathbb{E}\left[\Psi\left(Z_{T}^{t, s, z, \vartheta}-g\left(S_{T}^{t, s}\right)\right)\right] \geq p \text { for some } \vartheta \in \mathcal{U}(t, s, z)\right\},
$$

in which $\mathcal{U}(t, s, z)$ is the restriction to controls $\vartheta \in \mathcal{U}$ such that

$$
Z^{t, s, z, \vartheta} \geq-c_{\vartheta} \text { on }[t, T] \text { for some } c_{\vartheta} \geq 0 .
$$

Because this frictionless financial market is complete, one can describe $\pi$ explicitly under mild regularity and integrability conditions. We provide the proof of the following in the Appendix for completeness.

Proposition 2.2. Fix $(t, s, p) \in \mathfrak{D}$. Assume that the function $\Psi: \mathbb{R} \mapsto \operatorname{Im}(\Psi)$ is invertible, and that its inverse $\Phi$ is $C^{1}(\operatorname{Im}(\Psi))$. Assume further that $\Phi^{\prime}: \operatorname{Im}(\Psi) \rightarrow$ $(0, \infty)$ admits an inverse I. Finally assume that $\lambda^{t, s}:=(\mu / \sigma)\left(S^{t, s}\right)$ is square integrable and that the process $Q^{t, s}$ defined by

$$
Q^{t, s}:=\exp \left\{\frac{1}{2} \int_{t}\left|\lambda_{\tau}^{t, s}\right|^{2} d \tau+\int_{t}^{\cdot} \lambda_{\tau}^{t, s} d W_{\tau}\right\}
$$


satisfies

$$
\mathbb{E}\left[I\left(\hat{q} Q_{T}^{t, s}\right)\right]=p \quad \text { for some } \hat{q}>0,
$$

and

$$
g\left(S_{T}^{t, s}\right)+\Phi \circ I\left(\hat{q} Q_{T}^{t, s}\right) \in L^{1}\left(\mathbb{Q}^{t, s}\right) \text { where } d \mathbb{Q}^{t, s} / d \mathbb{P}=1 / Q_{T}^{t, s}
$$

Then,

$$
\pi(t, s, p)=\mathbb{E}^{\mathbb{Q}^{t, s}}\left[g\left(S_{T}^{t, s}\right)+\Phi \circ I\left(\hat{q} Q_{T}^{t, s}\right)\right] .
$$

As for the case with frictions, one can also obtain a characterization of $\pi$ in terms of a suitable Hamilton-Jacobi-Bellmann equation, see [12 and the Appendix. As in 53, it will be used to obtain an expansion of $v^{\epsilon}$ around $\epsilon=0$. We state it in terms of the function

$$
v:(t, s, p, x) \in \mathfrak{D} \times \mathbb{R} \mapsto \pi(t, s, p)-x,
$$

which is the analog of $v^{\epsilon}$ when the initial amount $x$ invested in the stock is non-zero. We note that formally $v^{0}$, obtained by setting $\epsilon$ to zero, is equal to $v$. In the following, we restrict to the case where $v$ is smooth, increasing and strictly convex in the parameter (the monotony and convexity just follow from the monotony and concavity of $\Psi$ ). A similar result in the sense of viscosity solutions can be found in [12].

Theorem 2.3. Assume that $\pi \in C^{1,2}\left(\mathfrak{D}_{<T}\right)$ and that $\min \left\{\pi_{p}, \pi_{p p}\right\}>0$ on $\mathfrak{D}_{<T}$. Then, $v(t, x, p, x)=\pi(t, s, p)-x$ is a strong solution of

$$
-\mathcal{L}_{S \theta} v-\hat{\mathcal{L}}_{P \mid S \theta} v=0 \text { on } \mathfrak{D}_{<T} \times \mathbb{R} \text { and } \Psi(v+x-g)=p \text { on } \mathfrak{D}_{T} \times \mathbb{R},
$$

where

$$
\begin{gathered}
\mathcal{L}_{P \mid S \theta}^{a} \varphi:=2^{-1} a\left(\bar{\sigma}_{\theta, a}+\bar{\sigma}_{\theta, 0}\right)^{\top} D \varphi_{p} \\
\hat{\mathcal{L}}_{P \mid S \theta} \varphi:=\inf \left\{\mathcal{L}_{P \mid S \theta}^{a} \varphi: a \in \mathbb{R} \text { s.t. } \bar{\sigma}_{\theta, a}^{\top} D \varphi=0\right\} \\
\mathcal{L}_{S \theta} \varphi:=\varphi_{t}+\bar{\mu}_{\theta}^{\top} D \varphi+\frac{1}{2} \operatorname{Tr}\left[\bar{\sigma}_{\theta, 0} \bar{\sigma}_{\theta, 0}^{\top} D^{2} \varphi\right]
\end{gathered}
$$

with, for $(t, s, p) \in \mathfrak{D}$,

$$
\begin{gathered}
\bar{\mu}_{\theta}(t, s):=\left(\begin{array}{c}
s \mu(t, s) \\
\theta(t, s, p) \mu(t, s) \\
0
\end{array}\right) \text { and } \bar{\sigma}_{\theta, a}(t, s, p):=\left(\begin{array}{c}
s \sigma(t, s) \\
\theta(t, s, p) \sigma(t, s) \\
a
\end{array}\right), \\
\theta(t, s, p)=\left(s \pi_{s}+\frac{\pi_{p}}{\sigma} \frac{\left(\frac{\mu}{\sigma} \pi_{p}-\sigma s \pi_{s p}\right)}{\pi_{p p}}\right)(t, s, p) .
\end{gathered}
$$

Remark 2.4. For later use, note that

$$
\hat{\mathcal{L}}_{\mathrm{P} \mid \mathrm{S} \theta} v=\mathcal{L}_{\mathrm{P} \mid \mathrm{S} \theta}^{\hat{a}} v \text { with } \hat{a}:=-\bar{\sigma}_{\theta, 0}^{\top} D v / v_{p}=\frac{\frac{\mu}{\sigma} v_{p}-\sigma s v_{p s}}{v_{p p}}
$$

and

$$
\theta=s \pi_{s}+\pi_{p} \hat{a} / \sigma
$$




\section{Small transaction costs expansion}

It follows from Proposition 2.2 that the value function $v$ associated to the frictionless case is known, or at least can be computed easily. Since it should identify to $v^{\epsilon}$ for $\epsilon=0$, we seek for an expansion of $v^{\epsilon}$ as $\epsilon \rightarrow 0$ in which $v$ is the 0 -order term. From [53, one can expect to obtain an $o\left(\epsilon^{2}\right)$-expansion if we introduce a second and a fourth order term, the last one depending on a fast variable, $\xi_{\epsilon}$ below. Namely, we seek for two functions $u$ and $\varpi$ such that

$$
v^{\epsilon}(\zeta, x)=v(\zeta, x)+\epsilon^{2} u(\zeta)+\epsilon^{4} \varpi \circ \xi_{\epsilon}(\zeta, x)+o\left(\epsilon^{2}\right) \text { for }(\zeta, x) \in \mathfrak{D} \times \mathbb{R}
$$

in which, for a map $w:(\zeta, \xi) \in \mathfrak{D} \times \mathbb{R} \mapsto w(\zeta, \xi)$, we set

$$
\left(w \circ \xi_{\epsilon}\right)(\zeta, x):=w\left(\zeta, \xi_{\epsilon}(\zeta, x)\right), \quad \text { with } \quad \xi_{\epsilon}(\zeta, x):=\frac{x-\theta(\zeta)}{\epsilon} .
$$

Note that when $w$ has sub quadratic growth in $\xi$, the term $\epsilon^{4} \varpi \circ \xi_{\epsilon}(\zeta, x)$ in (3.1) is in a lower order than $\epsilon^{2}$ and plays no role in the expansion. We will show that this is indeed the case. However, at least at the formal level, the second derivative of $\epsilon^{4} \varpi \circ \xi_{\epsilon}(\zeta, x)$ is exactly of order $\epsilon^{2}$ and this observation is crucial in deriving the corrector equations. Also, in the context of formal matched asymptotics, one may recognize (3.1) as in the inner expansion.

Remark 3.1. In the case where the domain of $\Psi$ is bounded from below, the convergence $v^{\epsilon} \rightarrow v$ can not hold except if $g$ is linear. Indeed, assume that the domain of $\Psi$ is bounded by $-\kappa \in \mathbb{R}$, i.e. $\Psi \equiv-\infty$ on $(-\infty,-\kappa)$. Then, it follows from [15] that $v^{\epsilon}(t, s, p, x) \geq \hat{g}(s)-x-\kappa$ for all $(t, s, p, x) \in \mathfrak{D}_{<T} \times \mathbb{R}$, where $\hat{g}$ is the concave envelope of $g$. On the other hand $\lim _{t \rightarrow T} v(t, s, p, x)=g(s)-x-\kappa+\Psi^{-1}(p)+\kappa$, by (2.11), where $\Psi^{-1}$ is the left-continuous inverse of $\Psi$. If $g$ is not concave, i.e. if $\{\hat{g}>g\}$ is non-empty, we therefore obtain that $v^{\epsilon}$ does not converges to $v$ on a non-empty subset of $\left\{(t, s, p) \in \mathfrak{D}_{<T}: \hat{g}(s)>g(s)+\Psi^{-1}(p)+\kappa\right\}$. Hence, we need to assume that $g$ is concave, i.e. $\hat{g} \equiv g$. It can actually neither be strictly concave on any interval of $(0, \infty)$. Otherwise, there will be $(t, s)$ such that $\mathbb{E}^{\mathbb{Q}^{t, s}}\left[g\left(S_{T}^{t, s}\right)\right]=: \bar{\pi}(t, s)<g(s)$ and therefore $v(t, s, p, x)<g(s)-x+\Psi^{-1}(p)=g(s)-x-\kappa+\Psi^{-1}(p)+\kappa$, since adding $-x+\Psi^{-1}(p)$ to $\bar{\pi}(t, s)$ allows to hedge $Z_{T}:=g\left(S_{T}^{t, s}\right)+\Psi^{-1}(p)$ which satisfies $\Psi\left(Z_{T}-g\left(S_{T}^{t, s}\right)\right)=p$. By choosing $p$ such that $\Psi^{-1}(p)+\kappa$ is close to 0 , we again obtain that $v^{\epsilon}(t, s, p, x)$ does not converge to $v(t, s, p, x)$ even if $\hat{g}=g$.

Our main result provides a precise characterization of the functions $u$ and $\varpi$ under the assumption that $v^{\epsilon}$ converges at a rate $O\left(\epsilon^{2}\right)$. We shall see that this is true in typical examples of application in Section 4 below.

Assumption 3.2. For any $\left(\zeta_{o}, x_{o}\right) \in \mathfrak{D} \times \mathbb{R}$, there exists $r_{o}, \epsilon_{o}>0$ such that

$$
\sup \left\{u^{\epsilon}(\zeta, x):=\frac{v^{\epsilon}(\zeta, x)-v(\zeta, x)}{\epsilon^{2}}, \quad(\zeta, x) \in B_{r_{o}}\left(\zeta_{o}, x_{o}\right) \cap(\mathfrak{D} \times \mathbb{R}), \epsilon \in\left(0, \epsilon_{o}\right]\right\}<\infty .
$$

\footnotetext{
${ }^{3}$ When the lower bound is zero, the boundary of the natural domain of the problem is given by the super-replication cost. We believe that in this case there is a boundary layer near this boundary.

${ }^{4}$ This assumption states that the expansion in the small parameter $\epsilon$ starts with a quadratic term. In other words, we assume that the order of proposed expansion is "correct". Under this and other regularity assumptions, we prove the expansion and derive formulae for the coefficients in the expansion. Indeed this assumption holds in many examples. However, in the case discussed in the Remark 3.1 we believe that there is a boundary layer and this assumption would only hold away from the superreplication cost.
} 
It allows us to give a sense to the relaxed semi-limits

$$
u^{*}(\zeta, x):=\limsup _{\epsilon \downarrow 0,\left(\zeta^{\prime}, x^{\prime}\right) \rightarrow(\zeta, x)} u^{\epsilon}\left(\zeta^{\prime}, x^{\prime}\right) \text { and } u_{*}(\zeta, x):=\liminf _{\epsilon \downarrow 0,\left(\zeta^{\prime}, x^{\prime}\right) \rightarrow(\zeta, x)} u^{\epsilon}\left(\zeta^{\prime}, x^{\prime}\right),
$$

which will be the main objects of our analysis. More precisely, we shall show that $u^{*}=u_{*}=: u$ does not depend on the $x$-variable and is a viscosity solution of

$$
\left\{\begin{array}{cc}
-\mathcal{H} \varphi-h=0 & \text { on } \mathfrak{D}_{<T}, \\
\varphi=0 & \text { on } \mathfrak{D}_{T},
\end{array}\right.
$$

where

$$
\mathcal{H} \varphi=\varphi_{t}+\frac{1}{2} \sigma^{2} s^{2} \varphi_{s s}+\frac{1}{2}(\hat{a})^{2} \varphi_{p p}+\sigma s \hat{a} \varphi_{s p}-\frac{\mu}{\sigma} \hat{a} \varphi_{p},
$$

in which $\hat{a}$ is defined in (2.14) , and $(\varpi, h)$ are the solution of the so-called first corrector equation, i.e. for each $(\zeta, \xi) \in \mathfrak{D}_{<T} \times \mathbb{R}$ :

$$
\max \left\{-\frac{1}{2}\left[\frac{\pi_{p p}}{\left(\pi_{p}\right)^{2}} \sigma^{2}\right](\zeta) \xi^{2}+h(\zeta)-\frac{1}{2}\left[\sigma^{2} \delta^{2}\right](\zeta) \varpi_{\xi \xi}(\zeta, \xi) ;-1+\varpi_{\xi}(\zeta, \xi) ;-1-\varpi_{\xi}(\zeta, \xi)\right\}=0,
$$

where

$$
\delta:=s \theta_{s}-\theta+\frac{\theta_{p}}{\pi_{p}}\left(\theta-s \pi_{s}\right) .
$$

In order to construct the pair $(\varpi, h)$, we need some smoothness and non-degeneracy conditions on the value function $\pi$ of the frictionless problem.

Assumption 3.3. The functions $\pi, \theta$ and $\delta$ are $C^{1,2}(\mathfrak{D})$ and $\left(\pi_{p p} \wedge \pi_{p} \wedge|\delta|\right)>0$ on $\mathfrak{D}$.

Lemma 3.4. Let the Assumption 3.3 hold. Then, there exists a locally bounded function $h$ on $\mathfrak{D}$ and a non-negative function $\varpi$ on $\mathfrak{D} \times \mathbb{R}$ such that, for all $\zeta \in \mathfrak{D}$, the map $\xi \in \mathbb{R} \mapsto \varpi(\zeta, \xi)$ is $C^{2}(\mathbb{R})$ and solves (3.7) on $\mathbb{R}$. Moreover, it satisfies

(i) $\varpi(\cdot, 0)=0$ on $\mathfrak{D}$.

(ii) $\varpi \in C^{1,2}(\mathfrak{D} \times \mathbb{R})$ and $\left|\varpi_{\xi}\right| \leq 1$ on $\mathfrak{D} \times \mathbb{R}$.

(iii) There exists a continuous function $\varrho: \mathfrak{D} \rightarrow \mathbb{R}$ such that

$$
\frac{|\varpi(\cdot, \xi)|}{1+|\xi|}+\left(\left|\varpi_{t}\right|+|D \varpi|+\left|D^{2} \varpi\right|\right)(\cdot, \xi) \leq \varrho \text { on } \mathfrak{D}, \forall \xi \in \mathbb{R} .
$$

(iv) There exists a continuous positive function $\hat{\xi}$ on $\mathfrak{D}$ such that, for all $(\zeta, \xi) \in \mathfrak{D} \times \mathbb{R}$,

$$
\varpi_{\xi}(\zeta, \xi)=-1 \Leftrightarrow \xi \leq-\hat{\xi}(\zeta) \text { and } \varpi_{\xi}(\zeta, \xi)=1 \Leftrightarrow \xi \geq \hat{\xi}(\zeta) .
$$

The proof of this result is postponed to Section 6. In that section, we also derive explicit expressions for $\varpi, h$ and $\hat{\xi}$ in terms of $\pi$ and its derivatives, see (6.5), (6.3) and (6.4) below.

Remark 3.5. It follows from Lemma 3.4 that we indeed have $|\varpi(\cdot, \xi)| \leq|\xi|$ for all $\xi \in \mathbb{R}$. This is a straightforward consequence of (3.7) and (i).

In order to fully characterize $u$ as $u^{*}=u_{*}$, we also need a comparison principle on (3.5). 
Assumption 3.6. There exists a set of functions $\mathcal{C}$ which contains $u^{*}$ and $u_{*}$, and such that $u_{1} \geq u_{2}$ on $\mathfrak{D}$ whenever $u_{1}$ (resp. $u_{2}$ ) is a lower semi-continuous (resp. upper semi-continuous) viscosity super-solution (resp. sub-solution) of (3.5) in $\mathcal{C}$.

Under the above conditions, we will prove in Section 5 that the expansion announced in (3.1) holds.

Theorem 3.7. Let the Assumptions 3.2, 3.3 and 3.6 hold. Then, (3.1) holds with $\varpi$ as in Lemma 3.4 and $u$ given by the unique viscosity solution of (3.5) in $\mathcal{C}$. Moreover, $u=u^{*}=u_{*}$.

Proof. This is an immediate consequence of Propositions 5.5 5.7 and 5.8 below, combined with Assumption 3.6

As explained above, the function $\pi$ is explicit or can be computed easily, and so is $v$, while $\varpi$ is given in (6.5) below in terms of $\pi$ and its derivatives. As for $u$, it solves the linear equation (3.5) which can be solved numerically whenever the function $\hat{a}$ defined in (2.14) and $\hat{a} \mu / \sigma$ are Lipschitz on $\mathfrak{D}$. Note that, in this case, it admits the Feynman-Kac representation

$$
u(t, s, p)=\mathbb{E}\left[\int_{t}^{T} h\left(\tau, \bar{S}_{\tau}^{t, s}, \bar{P}_{\tau}^{t, s, p}\right) d \tau\right],
$$

in which $\bar{S}^{t, s}$ solves (2.1) with $\mu \equiv 0$, and

$$
\bar{P}^{t, s, p}:=p-\int_{t}(\hat{a} \mu / \sigma)\left(\tau, \bar{S}_{\tau}^{t, s}, \bar{P}_{\tau}^{t, s, p}\right) d \tau+\int_{t} \hat{a}\left(\tau, \bar{S}_{\tau}^{t, s}, \bar{P}_{\tau}^{t, s, p}\right) d W_{\tau} .
$$

If the probability measure $\mathbb{Q}^{t, s}$ of Proposition 2.2 is well defined, this is equivalent to

$$
u(t, s, p)=\mathbb{E}^{\mathbb{Q}^{t, s}}\left[\int_{t}^{T} h\left(\tau, S_{\tau}^{t, s}, \hat{P}_{\tau}^{t, s, p}\right) d \tau\right]
$$

in which

$$
\hat{P}^{t, s, p}:=p+\int_{t}^{\cdot} \hat{a}\left(\tau, S_{\tau}^{t, s}, \hat{P}_{\tau}^{t, s, p}\right) d W_{\tau} .
$$

In the examples of Section 4 all these quantities are known, as far as one can compute the price and the greeks of a plain vanilla European option in the Black and Scholes model.

Note also that the functions $\pi$ and $\hat{\xi}$ can be used to construct almost optimal strategies in the original problem (2.7). This will be explained later on in Section 7 for the exponential and the power risk criterias.

Remark 3.8. We restrict here to the case of a single stock mainly for ease of notations. The arguments contained in Section 5 can essentially be reproduced in the multidimensional case. The main difficulties will come from the construction of $\varpi$ in Lemma 3.4 see [4], and from the existence of a solution to the Skorohod problem in the proofs of Section 7 .

\section{Examples}

In this section, we discuss two typical examples of application in which Assumptions 3.2 3.3 and 3.6 are satisfied, and therefore the expansion result of Theorem 3.7 can be applied. 


\subsection{The exponential risk criterion in the Black and Scholes model}

We first specialize the discussion to the case where the loss function $\Psi$ is of exponential form:

$$
\Psi(r):=-e^{-\eta r}, \quad r \in \mathbb{R}
$$

for some $\eta>0$, and the stock price $S^{t, s}$ follows the Black and Scholes dynamics

$$
S^{t, s}=s+\int_{t}^{\cdot} \lambda \sigma S_{\tau}^{t, s} d \tau+\int_{t}^{\cdot} \sigma S_{\tau}^{t, s} d W_{\tau}
$$

for some $(\lambda, \sigma) \in \mathbb{R} \times(0, \infty)$.

In this case, the pricing function $\pi$ can be derived explicitly. This is an easy consequence of Proposition 2.2. We recall that $h$ and $\hat{\xi}$ are given in (6.3) and (6.4) below.

Proposition 4.1. For all $(t, s, p) \in \mathfrak{D}:=[0, T] \times(0, \infty) \times(-\infty, 0)$,

$$
\pi(t, s, p)=\bar{\pi}(t, s)+\check{\pi}(t, p),
$$

where

$$
\check{\pi}(t, p):=-\frac{\lambda^{2}(T-t)}{2 \eta}-\frac{1}{\eta} \ln (-p)
$$

and

$$
\bar{\pi}(t, s):=\mathbb{E}^{\mathbb{Q}}\left[g\left(S_{T}^{t, s}\right)\right] \text { with } d \mathbb{Q} / d \mathbb{P}:=e^{-\frac{\lambda^{2}}{2} T-\lambda W_{T}} .
$$

Moreover, if $\bar{\pi} \in C^{0,2}([0, T] \times(0, \infty))$, then

$$
\left\{\begin{array}{c}
\theta(t, s)=s \bar{\pi}_{s}(t, s)+\frac{\lambda}{\sigma \eta}, \delta(t, s)=s^{2} \bar{\pi}_{s s}(t, s)-\frac{\lambda}{\sigma \eta}, \hat{a}(p)=-\lambda p \\
h(t, s)=\left(\frac{3}{16}\right)^{\frac{2}{3}} \sigma^{2} \eta^{\frac{1}{3}}|\delta(t, s)|^{\frac{4}{3}}, \hat{\xi}(t, s)=\left(\frac{3}{2 \eta}\right)^{\frac{1}{3}}|\delta(t, s)|^{\frac{2}{3}}
\end{array}\right.
$$

They are well-defined under the conditions of Assumption 4.2 below. Note in particular that

$$
\theta, \delta, h \text { and } \hat{\xi} \text { only depend on }(t, s) \text {. }
$$

Moreover, the second corrector equation (3.5) can be written as

$$
\left\{\begin{array}{cc}
-\varphi_{t}-\frac{1}{2} \sigma^{2} s^{2} \varphi_{s s}-\frac{\lambda^{2}}{2} p^{2} \varphi_{p p}+\sigma \lambda s p \varphi_{s p}+\lambda^{2} p \varphi_{p}-h=0 & \text { on } \mathfrak{D}_{<T} \\
\varphi=0 & \text { on } \mathfrak{D}_{T} .
\end{array}\right.
$$

If $h$ is bounded, which will be the case under Assumption 4.2 below, it follows from standard arguments that

$$
\hat{u}:(t, s) \in[0, T] \times(0, \infty) \mapsto \mathbb{E}^{\mathbb{Q}}\left[\int_{t}^{T} h\left(\tau, S_{\tau}^{t, s}\right) d \tau\right]
$$

is the unique viscosity solution of (4.6) in the class of functions having polynomial growth, see [18].

We now impose conditions under which Assumptions 3.2, 3.3 and 3.6 of Theorem 3.7 hold true. In particular, they are similar to the assumptions used in [5, Assumptions 3.1 and 3.2$] .5$

Assumption 4.2. The following holds:

\footnotetext{
${ }^{5}$ These assumptions can be verified directly using the frictionless equation and assumptions on $g$.
} 
a. $\bar{\pi} \in C^{1,4}(\mathfrak{D})$.

b. There exists $K>0$ such that

$$
|g|+\left|s \bar{\pi}_{s}\right|+\left|s^{2} \bar{\pi}_{s s}\right|+\left|\delta^{-1}\right|+\left|\theta_{t}\right|+\left|s^{2} \theta_{s s}\right| \leq K \quad \text { on } \mathfrak{D} .
$$

Note that these conditions imply in particular that $\hat{u}, \varpi \in C^{1,2}(\mathfrak{D})$, see (4.4) and (6.5) below for the exact expression of $\varpi$.

Proposition 4.3. Let $\Psi$ be as in (4.1) and $S$ as in (4.2). Then, Assumption 4.2 implies Assumptions 3.2. 3.3 and 3.6 of Theorem 3.7 .

The proof of this proposition is postponed to Section 7

Remark 4.4. [ $\epsilon^{2}$-optimal strategies] In the course of the proof of Proposition 4.3, we shall explain how to construct strategies which are optimal at the order $O\left(\epsilon^{2}\right)$, or $o\left(\epsilon^{2}\right)$ under an additional regularity assumption, for the problem with transaction costs, and which only depends on the knowledge of $v, \hat{u}, \varpi$ and $\theta$. See Propositions 7.1 and 7.2 below.

Note that, as a by-product, our expansion allows one to recover the result of [5] on the Hodges and Neuberger indifference price. More precisely, let $V^{\epsilon}$ be defined as

$$
V^{\epsilon}(t, s, y, x):=\sup _{L \in \mathfrak{L}^{\epsilon}(t, s, y, x)} \mathbb{E}\left[\Psi\left(Y_{T}^{t, y, \epsilon, L}+\ell^{\epsilon}\left(X_{T}^{t, x, s, L}\right)-g\left(S_{T}^{t, s}\right)\right)\right]
$$

and let $\tilde{V}^{\epsilon}$ be defined similarly but for $g \equiv 0$. Then, the indifference price associated to the market with transaction costs is given by

$$
q^{\epsilon}(t, s, y, x):=\inf \left\{q \in \mathbb{R}: V^{\epsilon}(t, s, y+q, x) \geq \tilde{V}^{\epsilon}(t, s, y, x)\right\} .
$$

It is easy to see that, for the exponential risk criterion, $q^{\epsilon}$ does not depend on the $y$-variable and that

$$
q^{\epsilon}(t, s, x)=-\frac{1}{\eta} \ln \left(\frac{\tilde{V}^{\epsilon}(t, s, y, x)}{V^{\epsilon}(t, s, y, x)}\right)=v^{\epsilon}(t, s,-1, x)-\tilde{v}^{\epsilon}(t, s,-1, x),
$$

in which $\tilde{v}^{\epsilon}$ is defined as $v^{\epsilon}$ but for $g \equiv 0$. Under the assumptions of Proposition 4.3 it then follows that

$$
q^{\epsilon}(t, s, x)=\bar{\pi}(t, s)+\epsilon^{2} \mathbb{E}^{\mathbb{Q}}\left[\int_{t}^{T} \Delta h\left(\tau, S_{\tau}^{t, s}\right) d \tau\right]+o\left(\epsilon^{2}\right),
$$

in which

$$
\Delta h(t, s):=\left(\frac{3}{16}\right)^{\frac{2}{3}} \sigma^{2} \eta^{\frac{1}{3}}\left(|\delta(t, s)|^{\frac{4}{3}}-\left|\frac{\lambda}{\sigma \eta}\right|^{\frac{4}{3}}\right) .
$$

\subsection{The power risk criterion in the Black and Scholes model}

We now consider the case

$$
\Psi(r):=-(r+\kappa)^{-\beta} \mathbf{1}_{\{r>\kappa\}}-\infty \mathbf{1}_{\{r \leq \kappa\}}, \quad r \in \mathbb{R},
$$

with $\beta, \kappa>0$. For this risk function, Proposition 2.2 implies that $\pi=\bar{\pi}+\hat{\pi}$ with

$$
\bar{\pi}(t, s)=\mathbb{E}^{\mathbb{Q}}\left[g\left(S_{T}^{t, s}\right)\right] \text { and } \hat{\pi}(t, p):=-\kappa+(-p)^{-\frac{1}{\beta}} m(t),
$$

for $(t, s, p) \in \mathfrak{D}$, in which $m$ is a $C_{b}^{1}([0, T])$ positive function satisfying $m(T)=1$. 
In view of Remark 3.1, we can however not expect to have $v^{\epsilon} \rightarrow v$ if $g$ is not linear. Since any linear payoff is hedged perfectly by the same buy-and-hold strategy in the two models, this boils down to considering the case $g \equiv 0$ up to an initial shift of $\kappa$ and $x$, at the costs of an additional $\epsilon^{3}$ term. We therefore restrict to the degenerate case $g \equiv 0$. Recall from Section 2.1 that the problem remains of interest, as $v^{\epsilon}$ is a building block for the analysis of optimal investment problems under risk constraints, see [10, 14.

Proposition 4.5. Let $\Psi$ be as in (4.8), $S$ as in (4.2) and $g \equiv 0$. Then Assumptions 3.2, 3.3 and 3.6 of Theorem 3.7 hold.

The proof is postponed to Section 7 .

Remark 4.6. [ $\epsilon^{2}$-optimal strategies] As in the exponential case, we produce in the course of the proof of Proposition 4.5 a strategy which is optimal at the order $o\left(\epsilon^{2}\right)$ for the problem with transaction costs, and which only depends on the knowledge of $v, \hat{u}$, $\varpi$ and $\theta$. See Remark 7.3 below.

\section{Derivation of the small transaction costs expansion}

\subsection{Preliminaries}

We start with the derivation of easy estimates that will be of important use in the sequel.

Remark 5.1. Observe that, for $(\zeta, x) \in \mathfrak{D} \times \mathbb{R}$, the initial dotation in cash and amount of stock $\left(v^{\epsilon}(\zeta, x)+x+\epsilon^{3}|x|, 0\right)$ can be turned into $\left(v^{\epsilon}(\zeta, x), x\right)$ by an immediate transfer $\Delta L_{0}=x$, while the initial dotation $\left(v^{\epsilon}(\zeta, 0)-x+\epsilon^{3}|x|, x\right)$ can be turned into $\left(v^{\epsilon}(\zeta, 0), 0\right)$ by an immediate transfer $\Delta L_{0}=-x$. By the definition of $v^{\epsilon}$, this implies that

$$
v^{\epsilon}(\zeta, 0)-\epsilon^{3}|x| \leq v^{\epsilon}(\zeta, x)+x \leq v^{\epsilon}(\zeta, 0)+\epsilon^{3}|x| .
$$

Remark 5.2. It follows from the same arguments as in [15, Proposition 6.1] that $v^{\epsilon} \geq v$.

Lemma 5.3. (i) The functions $u^{*}$ and $u_{*}$ are independent of the $x$-variable;

(ii) Moreover, for all $\zeta \in \mathfrak{D}$, we have

$$
u^{*}(\zeta)=\limsup _{\epsilon \downarrow 0, \zeta^{\prime} \rightarrow \zeta} u^{\epsilon *}\left(\zeta^{\prime}, \theta\left(\zeta^{\prime}\right)\right) \quad \text { and } \quad u_{*}(\zeta)=\liminf _{\epsilon \downarrow 0, \zeta^{\prime} \rightarrow \zeta} u_{*}^{\epsilon}\left(\zeta^{\prime}, \theta\left(\zeta^{\prime}\right)\right),
$$

in which $u^{\epsilon *}$ and $u_{*}^{\epsilon}$ denote the upper-and lower-semicontinuous envelopes of $u^{\epsilon}$.

Proof. We only show the result for $u^{*}$, the same reasoning can be used for the relaxed semi-limit $u_{*}$. Fix $\zeta \in \mathfrak{D}$ and $x \in \mathbb{R}$. By the definition of $u^{*}$, there exists a sequence $\left(\zeta_{\epsilon}, x_{\epsilon}\right)_{\epsilon>0}$ such that

$$
\left(\zeta_{\epsilon}, x_{\epsilon}\right) \underset{\epsilon \downarrow 0}{\longrightarrow}(\zeta, x) \text { and } u^{\epsilon}\left(\zeta_{\epsilon}, x_{\epsilon}\right) \underset{\epsilon \downarrow 0}{\longrightarrow} u^{*}(\zeta, x) .
$$

Fix also a sequence $\left(x_{\epsilon}^{\prime}\right)_{\epsilon>0}$ going to $x^{\prime} \in \mathbb{R}$ as $\epsilon \rightarrow 0$. By Remark 5.1 and the definitions of $u^{\epsilon}$ and $v$ in (3.3) and (2.10), we have

$$
\begin{aligned}
& v^{\epsilon}\left(\zeta_{\epsilon}, 0\right)-\epsilon^{3}\left|x_{\epsilon}\right| \leq \epsilon^{2} u^{\epsilon}\left(\zeta_{\epsilon}, x_{\epsilon}\right)+\pi\left(\zeta_{\epsilon}\right) \leq v^{\epsilon}\left(\zeta_{\epsilon}, 0\right)+\epsilon^{3}\left|x_{\epsilon}\right| \\
& v^{\epsilon}\left(\zeta_{\epsilon}, 0\right)-\epsilon^{3}\left|x_{\epsilon}^{\prime}\right| \leq \epsilon^{2} u^{\epsilon}\left(\zeta_{\epsilon}, x_{\epsilon}^{\prime}\right)+\pi\left(\zeta_{\epsilon}\right) \leq v^{\epsilon}\left(\zeta_{\epsilon}, 0\right)+\epsilon^{3}\left|x_{\epsilon}^{\prime}\right|
\end{aligned}
$$

so that

$$
-\epsilon\left(\left|x_{\epsilon}\right|+\left|x_{\epsilon}^{\prime}\right|\right) \leq u^{\epsilon}\left(\zeta_{\epsilon}, x_{\epsilon}\right)-u^{\epsilon}\left(\zeta_{\epsilon}, x_{\epsilon}^{\prime}\right) \leq \epsilon\left(\left|x_{\epsilon}\right|+\left|x_{\epsilon}^{\prime}\right|\right)
$$


Sending $\epsilon \rightarrow 0$ and using (5.3) then leads to

$$
\lim _{\epsilon \rightarrow 0} u^{\epsilon}\left(\zeta_{\epsilon}, x_{\epsilon}^{\prime}\right)=u^{*}(\zeta, x) .
$$

This shows in particular that $u^{*}\left(\zeta, x^{\prime}\right) \geq u^{*}(\zeta, x)$. By arbitrariness of $x, x^{\prime} \in \mathbb{R}$, the reverse inequality holds as well, showing that $u^{*}$ does not depend on its $x$-variable. Moreover, applied to $x=x^{\prime}:=\theta(\zeta)$ and $x_{\epsilon}^{\prime}:=\theta\left(\zeta_{\epsilon}\right)$, the above implies that

$$
\limsup _{\epsilon \downarrow 0, \zeta^{\prime} \rightarrow \zeta} u^{\epsilon *}\left(\zeta^{\prime}, \theta\left(\zeta^{\prime}\right)\right) \geq u^{*}(\zeta, \theta(\zeta))=\limsup _{\epsilon \downarrow 0,\left(\zeta^{\prime}, x^{\prime}\right) \rightarrow(\zeta, \theta(\zeta))} u^{\epsilon}\left(\zeta^{\prime}, x^{\prime}\right) .
$$

To conclude the proof of the left hand-side of (5.2), it remains to show that

$$
\limsup _{\epsilon \downarrow 0,\left(\zeta^{\prime}, x^{\prime}\right) \rightarrow(\zeta, \theta(\zeta))} u^{\epsilon}\left(\zeta^{\prime}, x^{\prime}\right)=\limsup _{\epsilon \downarrow 0,\left(\zeta^{\prime}, x^{\prime}\right) \rightarrow(\zeta, \theta(\zeta))} u^{\epsilon *}\left(\zeta^{\prime}, x^{\prime}\right),
$$

and to use the inequality

$$
\limsup _{\epsilon \downarrow 0,\left(\zeta^{\prime}, x^{\prime}\right) \rightarrow(\zeta, \theta(\zeta))} u^{\epsilon *}\left(\zeta^{\prime}, x^{\prime}\right) \geq \limsup _{\epsilon \downarrow 0, \zeta^{\prime} \rightarrow \zeta} u^{\epsilon *}\left(\zeta^{\prime}, \theta\left(\zeta^{\prime}\right)\right) .
$$

To see that the above holds, note that the continuity of $v$, see Assumption 3.3 and recall (2.10), implies that for $(\zeta, \xi) \in \mathfrak{D} \times \mathbb{R}$ and $\epsilon>0$ we can find $\left(\zeta_{\epsilon}, \xi_{\epsilon}\right) \in \mathfrak{D} \times \mathbb{R}$ such that $\left(v^{\epsilon}-v\right)(\zeta, \xi) \leq\left(v^{\epsilon *}-v\right)(\zeta, \xi) \leq\left(v^{\epsilon}-v\right)\left(\zeta_{\epsilon}, \xi_{\epsilon}\right)+\epsilon^{3}$. Recalling the definition of $u^{\epsilon}$ in (3.3), this proves (5.4).

In view of the above result, we shall from now on omit the $x$-variable in the functions $u^{*}$ and $u_{*}$.

\subsection{The key expansion lemma}

We now provide the following key lemma, which is the counterpart of 53 , Remark 3.4, Section 4.2].

Lemma 5.4. Assume that $\pi, \theta \in C^{1,2}\left(\mathfrak{D}_{<T}\right)$. For $\epsilon>0$, and two $C^{1,2}\left(\mathfrak{D}_{<T} \times \mathbb{R}\right)$ functions $\phi$ and $w$, define

$$
\psi^{\epsilon}=v+\epsilon^{2} \phi+\epsilon^{4} w^{\epsilon} \text { with } w^{\epsilon}:=w \circ \xi_{\epsilon} .
$$

Set $D_{\epsilon}^{\iota}:=\left(\mathfrak{D}_{<T} \times \mathbb{R}\right) \cap\left\{\psi_{p}^{\epsilon}>0\right\} \cap\left\{\epsilon^{2} \phi_{p}+\epsilon^{4} w_{p}^{\epsilon} \geq \iota \pi_{p}\right\}$ for some $\iota>-1$. Then,

$$
\epsilon^{-2}\left(\mathcal{L}_{S X}+\hat{\mathcal{L}}_{P \mid S X}\right) \psi^{\epsilon}=\frac{1}{2} \frac{\pi_{p p}}{\left(\pi_{p}\right)^{2}} \sigma^{2} \xi_{\epsilon}^{2}+\left(\mathcal{H}+\mathcal{L}_{X \mid S P}^{\hat{a}}\right) \phi+\frac{1}{2} \sigma^{2} \delta^{2}\left(w_{\xi \xi} \circ \xi_{\epsilon}\right)+R_{\epsilon} \text { on } D_{\epsilon}^{\iota},(5.6
$$

where

$$
\mathcal{L}_{X \mid S P}^{\hat{a}} \phi=\frac{1}{2} \sigma^{2} \theta^{2} \phi_{x x}+\sigma^{2} s \theta \phi_{s x}+\theta \sigma \hat{a} \phi_{p x}
$$

with $\hat{a}$ defined in (2.14), and where $R_{\epsilon}$ is a continuous map defined on $D_{\epsilon}^{\iota}$ such that:

(i) For each bounded set $B \subset D_{\epsilon}^{\iota}$, there exists $\epsilon_{B}>0$ such that $\left\{\epsilon^{-1} R_{\epsilon}(\zeta, x):(\zeta, x\right.$, $\left.\left.\xi_{\epsilon}(\zeta, x)\right) \in B, \epsilon \in\left(0, \epsilon_{B}\right]\right\}$ is bounded.

(ii) Let $B \subset D_{\epsilon}^{\iota}$ be a bounded set. Assume that $\phi \in C_{b}^{\infty}(B)$ and that $w$ satisfies (3.9). Then, there exists $\epsilon_{B}>0$ and $C_{B}>0$ such that

$$
\left|R_{\epsilon}(\zeta, x)\right| \leq C_{B}\left(1+\epsilon\left|\xi_{\epsilon}\right|+\epsilon^{2}\left|\xi_{\epsilon}\right|^{2}\right)(\zeta, x),
$$

for all $\epsilon \in\left(0, \epsilon_{B}\right]$ and $(\zeta, x) \in B$. 
Proof. All over this proof, we work on $D_{\epsilon}^{\iota}$ and omit the argument for simplicity.

Step 1: We first provide an expansion for $\mathcal{L}_{S X} \psi^{\epsilon}$. The first term follows from the relation $x=\theta+\epsilon \xi_{\epsilon}$ :

$$
\mathcal{L}_{\mathrm{SX}}\left(v+\epsilon^{2} \phi\right)=\mathcal{L}_{\mathrm{S} \theta}\left(v+\epsilon^{2} \phi\right)-\epsilon \mu \xi_{\epsilon}+\epsilon^{2} R_{1}^{\epsilon},
$$

with

$$
R_{1}^{\epsilon}=\epsilon \xi_{\epsilon}\left(\mu \phi_{x}+\frac{\sigma^{2}}{2}\left(\left(2 \theta+\epsilon \xi_{\epsilon}\right) \phi_{x x}+2 s \phi_{x s}\right)\right) .
$$

Then, we use the fact that $\xi_{\epsilon}=\xi_{1} / \epsilon$ and the definitions of $\bar{\sigma}_{a}$ and $\bar{\sigma}_{\theta, a}$ in (2.8) and (2.12) to obtain

$$
\begin{aligned}
\mathcal{L}_{\mathrm{SX}}\left(\epsilon^{4} w^{\epsilon}\right) & =\frac{\epsilon^{2}}{2}\left(w_{\xi \xi} \circ \xi_{\epsilon}\right) D \xi_{1}^{\top} \bar{\sigma}_{0} \bar{\sigma}_{0}^{\top} D \xi_{1}+\epsilon^{2} R_{2}^{\epsilon} \\
& =\frac{\epsilon^{2}}{2}\left(w_{\xi \xi} \circ \xi_{\epsilon}\right) D \xi_{1}^{\top} \bar{\sigma}_{\theta, 0} \bar{\sigma}_{\theta, 0}^{\top} D \xi_{1}+\epsilon^{2} R_{3}^{\epsilon},
\end{aligned}
$$

where

$$
R_{2}^{\epsilon}=\epsilon^{2}\left(\mathcal{L}_{\mathrm{S}} w\right) \circ \xi_{\epsilon}+\epsilon\left(\left(w_{\xi} \circ \xi_{\epsilon}\right) \mathcal{L}_{\mathrm{SX}} \xi_{1}+2 s^{2} \sigma^{2} \partial_{s} \xi_{1}\left(w_{s \xi} \circ \xi_{\epsilon}\right)\right)
$$

and

$$
\begin{aligned}
R_{3}^{\epsilon} & =R_{2}^{\epsilon}+\frac{\sigma^{2}}{2}\left(w_{\xi \xi} \circ \xi_{\epsilon}\right)\left(D_{(s, x)} \xi_{1}\right)^{\top}\left(\begin{array}{cc}
0 & s \epsilon \xi_{\epsilon} \\
s \epsilon \xi_{\epsilon} & \theta \epsilon \xi_{\epsilon}+\left(\epsilon \xi_{\epsilon}\right)^{2}
\end{array}\right) D_{(s, x)} \xi_{1} \\
& =R_{2}^{\epsilon}+\epsilon \xi_{\epsilon} \frac{\sigma^{2}}{2}\left(w_{\xi \xi} \circ \xi_{\epsilon}\right)\left(D_{(s, x)} \xi_{1}\right)^{\top}\left(\begin{array}{cc}
0 & s \\
s & \theta+\epsilon \xi_{\epsilon}
\end{array}\right) D_{(s, x)} \xi_{1} .
\end{aligned}
$$

Combining the above expansions leads to

$$
\mathcal{L}_{\mathrm{SX}} \psi^{\epsilon}=\mathcal{L}_{\mathrm{S} \theta}\left(v+\epsilon^{2} \phi\right)-\epsilon \mu \xi_{\epsilon}+\frac{\epsilon^{2}}{2}\left(w_{\xi \xi} \circ \xi_{\epsilon}\right) D \xi_{1}^{\top} \bar{\sigma}_{\theta, 0} \bar{\sigma}_{\theta, 0}^{\top} D \xi_{1}+\epsilon^{2}\left(R_{1}^{\epsilon}+R_{3}^{\epsilon}\right)
$$

Step 2: We now focus on the operator $\hat{\mathcal{L}}_{P \mid S X}$ applied to $\psi^{\epsilon}$. Since $\psi_{p}^{\epsilon}>0$ on $D_{\epsilon}^{\iota}$, we have

$$
\hat{\mathcal{L}}_{\mathrm{P} \mid \mathrm{SX}} \psi^{\epsilon}=\mathcal{L}_{\mathrm{P} \mid \mathrm{SX}}^{a^{\epsilon}} \psi^{\epsilon} \text { with } a^{\epsilon}:=\frac{-\bar{\sigma}_{0}^{\top} D \psi^{\epsilon}}{\pi_{p}} \times \frac{1}{1+\epsilon^{2} \partial_{p}\left(\phi+\epsilon^{2} w^{\epsilon}\right) / \pi_{p}} .
$$

a. We first provide an expansion for $a^{\epsilon}$ around $\hat{a}$ defined in (2.14). We start by performing a first order expansion on the right-hand side of (5.8) to obtain

$$
a^{\epsilon}=\frac{-\bar{\sigma}_{0}^{\top} D \psi^{\epsilon}}{\pi_{p}} \times\left(1-\epsilon^{2} \partial_{p}\left(\phi+\epsilon^{2} w^{\epsilon}\right) / \pi_{p}\right)+R_{4}^{\epsilon},
$$

where $R_{4}^{\epsilon}$ is a continuous map satisfying

$$
\begin{aligned}
\left|R_{4}^{\epsilon}\right| & \leq \frac{\left|\bar{\sigma}_{0}^{\top} D \psi^{\epsilon}\right|}{\pi_{p}} \frac{2}{(1+\iota)^{3}}\left|\epsilon^{2} \partial_{p}\left(\phi+\epsilon^{2} w^{\epsilon}\right) / \pi_{p}\right|^{2} \quad \text { on } D_{\epsilon}^{\iota} \\
& =\frac{\left|\bar{\sigma}_{0}^{\top} D \psi^{\epsilon}\right|}{\pi_{p}} \frac{2}{(1+\iota)^{3}}\left|\epsilon^{2} \frac{\phi_{p}}{\pi_{p}}-\epsilon^{3} \frac{\theta_{p}\left(w_{\xi} \circ \xi_{\epsilon}\right)}{\pi_{p}}+\epsilon^{4} \frac{\left(w_{p} \circ \xi_{\epsilon}\right)}{\pi_{p}}\right|^{2} .
\end{aligned}
$$

Then, we obverse that

$$
\begin{aligned}
-\bar{\sigma}_{0}^{\top} D \psi^{\epsilon} & =-\bar{\sigma}_{0}^{\top} D v-\bar{\sigma}_{0}^{\top} D\left(\epsilon^{2} \phi+\epsilon^{4} w^{\epsilon}\right) \\
& =-\bar{\sigma}_{\theta, 0}^{\top} D v+\sigma \epsilon \xi_{\epsilon}-\bar{\sigma}_{\theta+\epsilon \xi_{\epsilon}, 0} D\left(\epsilon^{2} \phi+\epsilon^{4} w^{\epsilon}\right) .
\end{aligned}
$$


By the definition of $\hat{a}$ in (2.14), dividing the above by $\pi_{p}=v_{p}$ implies

$$
\frac{-\bar{\sigma}_{0}^{\top} D \psi^{\epsilon}}{\pi_{p}}=\hat{a}+\epsilon \frac{\sigma \xi_{\epsilon}}{\pi_{p}}-\frac{\bar{\sigma}_{\theta+\epsilon \xi_{\epsilon}, 0}^{\top} D\left(\epsilon^{2} \phi+\epsilon^{4} w^{\epsilon}\right)}{\pi_{p}} .
$$

Recalling (5.9), this leads to

$$
\begin{aligned}
a^{\epsilon} & =\hat{a}+\epsilon \frac{\sigma \xi_{\epsilon}}{\pi_{p}}-\epsilon^{2} \frac{\bar{\sigma}_{\theta, \hat{a}}^{\top} D \phi}{\pi_{p}}+R_{5}^{\epsilon}, \\
\left(a^{\epsilon}\right)^{2} & =(\hat{a})^{2}+2 \epsilon \hat{a} \frac{\sigma \xi_{\epsilon}}{\pi_{p}}+\epsilon^{2}\left[\left(\frac{\sigma \xi_{\epsilon}}{\pi_{p}}\right)^{2}-2 \hat{a} \bar{\sigma}_{\theta, \hat{a}}^{\top} D \phi / \pi_{p}\right]+R_{6}^{\epsilon},
\end{aligned}
$$

where

$$
\begin{aligned}
R_{5}^{\epsilon}:= & R_{4}^{\epsilon}-\epsilon^{2}\left[\epsilon \xi_{\epsilon} \frac{\sigma \phi_{p}}{\left(\pi_{p}\right)^{2}}+\frac{\bar{\sigma}_{\epsilon \xi_{\epsilon}, 0}^{\top} D \phi}{\pi_{p}}\right]+\epsilon^{4} \frac{w_{p}^{\epsilon}}{\pi_{p}}\left(-\hat{a}-\epsilon \xi_{\epsilon} \frac{\sigma}{\pi_{p}}+\epsilon^{2} \frac{\bar{\sigma}_{\theta+\epsilon \xi_{\epsilon}, 0}^{\top} D \phi}{\pi_{p}}\right) \\
& -\epsilon^{4} \frac{\bar{\sigma}_{\theta+\epsilon \xi_{\epsilon}, 0}^{\top} D w^{\epsilon}}{\pi_{p}}\left(1-\epsilon^{2} \partial_{p}\left(\phi+\epsilon^{2} w^{\epsilon}\right) / \pi_{p}\right)+\epsilon^{4} \frac{\phi_{p}}{\pi_{p}^{2}} \bar{\sigma}_{\theta+\epsilon \xi_{\epsilon}, 0}^{\top} D \phi, \\
R_{6}^{\epsilon}= & \left(-\epsilon^{2} \frac{\bar{\sigma}_{\theta, \hat{a}}^{\top} D \phi}{\pi_{p}}+R_{5}^{\epsilon}\right)^{2}+2 \hat{a} R_{5}^{\epsilon}+2 \frac{\sigma \xi_{\epsilon}}{\pi_{p}}\left(-\epsilon^{2} \frac{\bar{\sigma}_{\theta, \hat{a}}^{\top} D \phi}{\pi_{p}}+R_{5}^{\epsilon}\right) .
\end{aligned}
$$

b. We now plug the expansions (5.10) and (5.11) in the left-hand side equality in (5.8) to obtain

$$
\begin{aligned}
\hat{\mathcal{L}}_{\mathrm{P} \mid \mathrm{SX}} \psi^{\epsilon}= & \mathcal{L}_{\mathrm{P} \mid \mathrm{S} \theta}^{\hat{a}} v+\epsilon\left[\pi_{p p} \hat{a} \frac{\sigma \xi_{\epsilon}}{\pi_{p}}+\sigma^{2} s \pi_{s p} \frac{\xi_{\epsilon}}{\pi_{p}}\right] \\
& +\epsilon^{2}\left(\frac{1}{2} \pi_{p p}\left[\left(\frac{\sigma \xi_{\epsilon}}{\pi_{p}}\right)^{2}-2 \hat{a}\left(\frac{\bar{\sigma}_{\theta, \hat{a}}^{\top} D \phi}{\pi_{p}}\right)\right]-\sigma s \pi_{s p} \frac{\bar{\sigma}_{\theta, \hat{a}}^{\top} D \phi}{\pi_{p}}+\mathcal{L}_{\mathrm{P} \mid \mathrm{S} \theta}^{\hat{a}} \phi\right) \\
& +\epsilon^{2}\left(\frac{1}{2}\left(w_{\xi \xi} \circ \xi_{\epsilon}\right) D \xi_{1}^{\top}\left(\bar{\sigma}_{\theta, \hat{a}} \bar{\sigma}_{\theta, \hat{a}}^{\top}-\bar{\sigma}_{\theta, 0} \bar{\sigma}_{\theta, 0}^{\top}\right) D \xi_{1}+R_{7}^{\epsilon}\right)
\end{aligned}
$$

with

$$
\begin{aligned}
R_{7}^{\epsilon}= & \frac{1}{2} R_{6}^{\epsilon} \pi_{p p}+R_{5}^{\epsilon} \sigma s \pi_{s p}+\frac{1}{2}\left(\left(a^{\epsilon}\right)^{2}-(\hat{a})^{2}\right)\left(\epsilon^{2} \phi_{p p}+\epsilon^{4} w_{p p}^{\epsilon}\right)+\sigma s\left(a^{\epsilon}-\hat{a}\right)\left(\epsilon^{2} \phi_{s p}+\epsilon^{4} w_{s p}^{\epsilon}\right) \\
& +\left[\left(a^{\epsilon}-\hat{a}\right)\left(\epsilon \xi_{\epsilon}+\theta\right)+\hat{a} \epsilon \xi_{\epsilon}\right] \sigma \phi_{p x} \\
& +\frac{\epsilon^{3}}{2}(\hat{a})^{2}\left(\epsilon w_{p p}-2 \theta_{p} w_{p \xi}-\theta_{p p} w_{\xi}\right) \circ \xi_{\epsilon} \\
& +\epsilon^{3} \hat{a} \sigma\left(\epsilon s w_{s p}-s \theta_{p} w_{s \xi}-s \theta_{s} w_{p \xi}-s \theta_{s p} w_{\xi}+\theta w_{p \xi}\right) \circ \xi_{\epsilon} .
\end{aligned}
$$

Step 3: It remains to combine the results of Steps 1 and 2. We first observe that (2.11) and the definition $\hat{a}$ implies that

$$
\mathcal{L}_{\mathrm{S} \theta} v+\mathcal{L}_{\mathrm{P} \mid \mathrm{S} \theta}^{\hat{a}} v=\mathcal{L}_{\mathrm{S} \theta} v+\hat{\mathcal{L}}_{\mathrm{P} \mid \mathrm{S} \theta} v=0 .
$$

Second, we use (2.14) and the identity $v=\pi-x$ to obtain

$$
\hat{a}=\frac{\frac{\mu}{\sigma} \pi_{p}-\sigma s \pi_{p s}}{\pi_{p p}},
$$

which leads to

$$
\epsilon \xi_{\epsilon}\left(-\mu+\pi_{p p} \hat{a} \frac{\sigma}{\pi_{p}}+\sigma^{2} s \pi_{s p} \frac{1}{\pi_{p}}\right)=0
$$


and

$$
\begin{aligned}
\mathcal{L}_{\mathrm{S} \theta} \phi+\mathcal{L}_{\mathrm{P} \mid \mathrm{S} \theta}^{\hat{a}} \phi-\pi_{p p} \hat{a}\left(\frac{\bar{\sigma}_{\theta, \hat{a}}^{\top} D \phi}{\pi_{p}}\right)-\sigma s \pi_{s p} \frac{\bar{\sigma}_{\theta, \hat{a}}^{\top} D \phi}{\pi_{p}} & =\mathcal{L}_{\mathrm{S} \theta} \phi+\mathcal{L}_{\mathrm{P} \mid \mathrm{S} \theta}^{\hat{a}} \phi-\frac{\mu}{\sigma} \bar{\sigma}_{\theta, \hat{a}}^{\top} D \phi \\
& =\left(\mathcal{H}+\mathcal{L}_{\mathrm{X} \mid \mathrm{SP}}^{\hat{a}}\right) \phi .
\end{aligned}
$$

Finally, we use the identities $\xi_{1}=\theta-x$ and $\hat{a}=\left(\theta-s \pi_{s}\right) \sigma / \pi_{p}$, recall (2.15), to obtain

$$
\sigma^{2} \delta^{2}=D \xi_{1}^{\top} \bar{\sigma}_{\theta, 0} \bar{\sigma}_{\theta, 0}^{\top} D \xi_{1}+D \xi_{1}^{\top}\left(\bar{\sigma}_{\theta, \hat{a}} \bar{\sigma}_{\theta, \hat{a}}^{\top}-\bar{\sigma}_{\theta, 0} \bar{\sigma}_{\theta, 0}^{\top}\right) D \xi_{1},
$$

where $\delta$ is defined in (3.8). The above identities combined with (5.7) and (5.12) leads to (5.6) for $R_{\epsilon}$ defined as

$$
R_{\epsilon}:=R_{1}^{\epsilon}+R_{3}^{\epsilon}+R_{7}^{\epsilon}
$$

Step 4: The estimates on $R_{\epsilon}$ follow from direct computations.

\subsection{Viscosity subsolution property}

Proposition 5.5. Let the conditions of Theorem 3.7 hold. Then, $u^{*}$ is a viscosity subsolution of (3.5).

Proof. Let $\zeta_{o} \in \mathfrak{D}_{<T}$ and $\varphi \in C^{1,2}\left(\mathfrak{D}_{<T}\right)$ be such that

$$
\max _{\mathfrak{D}<T}(\text { strict })\left(u^{*}-\varphi\right)=\left(u^{*}-\varphi\right)\left(\zeta_{o}\right) .
$$

By Lemma 5.3, there exists $\left(\zeta^{\epsilon}\right)_{\epsilon>0}$ satisfying

$$
\begin{gathered}
\zeta^{\epsilon} \underset{\epsilon \downarrow 0}{\longrightarrow} \zeta_{o}, \quad x^{\epsilon}:=\theta\left(\zeta^{\epsilon}\right) \underset{\epsilon \downarrow 0}{\longrightarrow} \theta\left(\zeta_{o}\right)=: x_{o}, \\
u^{\epsilon *}\left(\zeta^{\epsilon}, x^{\epsilon}\right) \underset{\epsilon \downarrow 0}{\longrightarrow} u^{*}\left(\zeta_{o}\right) \text { and } \Delta_{\epsilon}:=u^{\epsilon *}\left(\zeta^{\epsilon}, x^{\epsilon}\right)-\varphi\left(\zeta^{\epsilon}\right) \underset{\epsilon \downarrow 0}{\longrightarrow} 0 .
\end{gathered}
$$

Assumptions 3.2 and 3.3 entail the existence of $\bar{r}_{o}>0,0<r_{o} \leq \bar{r}_{o}$ and $\epsilon_{o}>0$ such that

$$
\bar{m}:=\sup \left\{u^{\epsilon *}(\zeta, x),(\zeta, x) \in B_{o}, \epsilon \in\left(0, \epsilon_{o}\right]\right\}<\infty,
$$

and

$$
\theta \in \bar{B}_{\frac{\bar{x}_{o}}{4}}\left(x_{o}\right) \quad \text { on } \quad \bar{B}_{r_{o}}\left(\zeta_{o}\right),
$$

where $B_{o}:=B_{r_{o}}\left(\zeta_{o}\right) \times B_{\bar{r}_{o}}\left(x_{o}\right)$. After possibly changing $\epsilon_{o}$, we can also assume that

$$
\left|\zeta^{\epsilon}-\zeta_{o}\right| \vee\left|x^{\epsilon}-x_{o}\right| \leq \frac{r_{o}}{4} \quad \text { and } \quad\left|\Delta_{\epsilon}\right| \leq 1 \quad \text { for all } \epsilon \in\left(0, \epsilon_{o}\right]
$$

We have

$$
u^{\epsilon *} \leq \bar{m} \quad \text { on } B_{o} \quad \text { for } \quad \epsilon \in\left(0, \epsilon_{o}\right],
$$

and, by Assumption 3.3 .

$$
\pi_{p p} \wedge \pi_{p}>\iota \text { on } B_{r_{o}}\left(\zeta_{o}\right), \text { for some } \iota \in(0,1) .
$$

Step 1: We first construct a suitable test function for $v^{\epsilon}$, for $\epsilon \in\left(0, \epsilon_{o}\right]$.

Since the function $\varphi$ is continuous,

$$
\sup \left\{2+\bar{m}-\varphi(\zeta) ; \zeta \in \bar{B}_{r_{o}}\left(\zeta_{o}\right)\right\}=: \bar{M}<+\infty .
$$

On the other hand, (5.16) implies that there is $\gamma>0$ such that

$$
\left|\zeta-\zeta^{\epsilon}\right|^{4} \geq \gamma \quad \text { for } \quad \zeta \in \bar{B}_{r_{o}}\left(\zeta_{o}\right) \backslash \bar{B}_{\frac{r_{o}}{2}}\left(\zeta_{o}\right) .
$$


We choose a strictly non-negative constant $c_{o}$ satisfying $c_{o}\left(\gamma \wedge\left(\frac{r_{o}}{4}\right)^{4}\right) \geq \bar{M}$ and define for $\epsilon \in(0,1)$

$$
\phi^{\epsilon}:(\zeta, x) \in \mathfrak{D} \times \mathbb{R} \mapsto c_{o}\left(\left|\zeta-\zeta^{\epsilon}\right|^{4}+|x-\theta(\zeta)|^{4}\right) .
$$

Consider now the following subset of $\bar{B}_{o}$ :

$$
B_{o, \frac{1}{2}}:=\left\{(\zeta, x) \in \bar{B}_{o} \text { s.t. } \zeta \in \bar{B}_{\frac{r_{o}}{2}}\left(\zeta_{o}\right) \text { and } x \in \bar{B}_{\frac{\bar{r}_{o}}{2}}\left(x_{o}\right)\right\} .
$$

It follows from (5.19), (5.15) and the choice of $c_{o}$ that

$$
\phi^{\epsilon} \geq 2+\bar{m}-\varphi \quad \text { on } \bar{B}_{o} \backslash B_{o, \frac{1}{2}} .
$$

We now define, for $\eta \in(0,1]$,

$$
\psi^{\epsilon, \eta}:=v+\epsilon^{2}\left(\Delta_{\epsilon}+\varphi+\phi^{\epsilon}\right)+\epsilon^{4}(1+\eta) \varpi \circ \xi_{\epsilon},
$$

where the function $\xi_{\epsilon}$ is defined in (3.2) and $\varpi$ is given in Lemma 3.4.

Step 2: Given $\epsilon \in\left(0, \epsilon_{o}\right]$ and $\eta \in(0,1]$, we now show that $v^{\epsilon *}-\psi^{\epsilon, \eta}$ admits a local maximizer $\left(\tilde{\zeta}^{\epsilon}, \tilde{x}^{\epsilon}\right)$ in $B_{0}$.

Note that, a-priori, this local maximizer should depend on $\eta$. We shall not emphasize this to alleviate notations but will come back to this point at the end of the proof. We set

$$
I^{\epsilon, \eta}:=u^{\epsilon *}-\Delta_{\epsilon}-\varphi-\phi^{\epsilon}-\epsilon^{2}(1+\eta) \varpi \circ \xi_{\epsilon} .
$$

Combining the fact that $\varpi(\cdot, 0)=0$, see Lemma 3.4 (5.16) and the definitions of $x^{\epsilon}$, $\Delta_{\epsilon}$ and $\phi^{\epsilon}$, we obtain

$$
\sup _{\bar{B}_{o}} I^{\epsilon, \eta} \geq \sup _{\bar{B}_{o, \frac{1}{2}}} I^{\epsilon, \eta} \geq I^{\epsilon, \eta}\left(\zeta^{\epsilon}, x^{\epsilon}\right)=0 .
$$

On the other hand, by (5.16), (5.17), (5.20), the fact that $\varpi \geq 0$, see Lemma 3.4, and the defnition of $\bar{m}$, we have

$$
I^{\epsilon, \eta} \leq u^{\epsilon *}-\bar{m}-1-\epsilon^{2}(1+\eta) \varpi \circ \xi_{\epsilon}<0 \quad \text { on } \quad \bar{B}_{o} \backslash \bar{B}_{o, \frac{1}{2}},
$$

after possibly changing $\epsilon_{0}$. Also $I^{\epsilon, \eta}$ is upper-semicontinuous. Hence, we may find a $\operatorname{maximizer}\left(\tilde{\zeta}^{\epsilon}, \tilde{x}^{\epsilon}\right) \in \bar{B}_{o, \frac{1}{2}} \subset B_{o}$ which satisfies

$$
I^{\epsilon, \eta}\left(\tilde{\zeta}^{\epsilon}, \tilde{x}^{\epsilon}\right) \geq 0 \text { and } \quad\left|\epsilon \xi_{\epsilon}\left(\tilde{\zeta}^{\epsilon}, \tilde{x}^{\epsilon}\right)\right| \vee\left|\tilde{\zeta}^{\epsilon}-\zeta_{o}\right| \leq r_{1},
$$

for some constant $r_{1}>0$. We recall that

$$
\epsilon \xi_{\epsilon}\left(\tilde{\zeta}^{\epsilon}, \tilde{x}^{\epsilon}\right)=\tilde{x}^{\epsilon}-\theta\left(\tilde{\zeta}^{\epsilon}\right) .
$$

Step 3: We now prove that there exists $\bar{\epsilon}_{o} \leq \epsilon_{o}$ such that for all $\epsilon \in\left(0, \bar{\epsilon}_{o}\right]$ we have

$$
-\hat{\xi}\left(\tilde{\zeta}^{\epsilon}\right)<\xi_{\epsilon}\left(\tilde{\zeta}^{\epsilon}, \tilde{x}^{\epsilon}\right)<\hat{\xi}\left(\tilde{\zeta}^{\epsilon}\right)
$$

where $\hat{\xi}$ is given in Lemma 3.4

We only prove the right hand-side. The other inequality is proved similarly. We first observe that Theorem 2.1 and step 2 imply that

$$
-\epsilon^{3}+1+\psi_{x}^{\epsilon, \eta}\left(\tilde{\zeta}^{\epsilon}, \tilde{x}^{\epsilon}\right) \leq 0 .
$$

Recalling the definitions of $\psi^{\epsilon, \eta}, v$ and $\phi^{\epsilon}$, direct computations lead to

$$
1+\psi_{x}^{\epsilon, \eta}\left(\tilde{\zeta}^{\epsilon}, \tilde{x}^{\epsilon}\right)=4 \epsilon^{2} c_{o}\left(\epsilon \xi_{\epsilon}\left(\tilde{\zeta}^{\epsilon}, \tilde{x}^{\epsilon}\right)\right)^{3}+\epsilon^{3}(1+\eta) \varpi_{\xi} \circ \xi_{\epsilon}\left(\tilde{\zeta}^{\epsilon}, \tilde{x}^{\epsilon}\right),
$$


so that we may rewrite (5.24) as

$$
-\epsilon+\epsilon(1+\eta) \varpi_{\xi} \circ \xi_{\epsilon}\left(\tilde{\zeta}^{\epsilon}, \tilde{x}^{\epsilon}\right) \leq-4 c_{o}\left(\epsilon \xi_{\epsilon}\left(\tilde{\zeta}^{\epsilon}, \tilde{x}^{\epsilon}\right)\right)^{3} .
$$

Assume now that the right hand-side of (5.23) does not hold for all $\epsilon>0$, small enough. Then, there exists a sequence $\left(\epsilon_{k}\right)_{k \geq 1}$ satisfying $\epsilon_{k} \rightarrow 0$ as $k \rightarrow \infty$ such that

$$
\xi_{\epsilon_{k}}\left(\tilde{\zeta}^{\epsilon_{k}}, \tilde{x}^{\epsilon_{k}}\right) \geq \hat{\xi}\left(\tilde{\zeta}^{\epsilon_{k}}\right) .
$$

Recall from Lemma 3.4 that this implies that

$$
\varpi_{\xi} \circ \xi_{\epsilon_{k}}\left(\tilde{\zeta}^{\epsilon_{k}}, \tilde{x}^{\epsilon_{k}}\right)=1 \text { and } \xi_{\epsilon_{k}}\left(\tilde{\zeta}^{\epsilon_{k}}, \tilde{x}^{\epsilon_{k}}\right)>0 .
$$

Combined with (5.25) the later leads to a contradiction since $c_{o}, \eta, \epsilon_{k}>0$.

Step 4: We now prove that there is $\bar{\xi} \in \mathbb{R}$ such that

$$
0 \geq\left(-\frac{1}{2} \frac{\pi_{p p}}{\left(\pi_{p}\right)^{2}} \sigma^{2} \bar{\xi}^{2}-\mathcal{H} \varphi-\frac{1}{2} \sigma^{2} \delta^{2}(1+\eta) \varpi_{\xi \xi}(\cdot, \bar{\xi})\right)\left(\zeta_{o}\right)
$$

Recall that $\hat{\xi}$ is a continuous functions. In view of (5.23) and (5.21), it follows that

$$
\left(\tilde{\zeta}^{\epsilon}, \tilde{x}^{\epsilon}, \xi_{\epsilon}\left(\tilde{\zeta}^{\epsilon}, \tilde{x}^{\epsilon}\right)\right)_{0<\epsilon \leq \bar{\epsilon}_{o}} \text { is bounded. }
$$

We can then find a sequence $\left(\epsilon_{n}\right)_{n \geq 1} \subset\left(0, \bar{\epsilon}_{o}\right]$ such that $\epsilon_{n} \rightarrow 0$ as $n \rightarrow \infty$ and

$$
\left(\tilde{\zeta}^{\epsilon_{n}}, \tilde{x}^{\epsilon_{n}}, \xi_{\epsilon_{n}}\left(\tilde{\zeta}^{\epsilon_{n}}, \tilde{x}^{\epsilon_{n}}\right)\right) \rightarrow(\bar{\zeta}, \bar{x}, \bar{\xi}) \in \mathfrak{D} \times \mathbb{R} \times \mathbb{R} \text { as } n \rightarrow \infty .
$$

Moreover, classical arguments show that

$$
(\bar{\zeta}, \bar{x})=\left(\zeta_{o}, x_{o}\right)
$$

Observe for later use that

$$
-\hat{\xi}\left(\zeta_{o}\right) \leq \bar{\xi} \leq \hat{\xi}\left(\zeta_{o}\right)
$$

by (5.23) and the continuity of $\hat{\xi}$. By Step 2 and Theorem 2.1 again, we have

$$
\left\{-\mathcal{L}_{\mathrm{SX}} \psi^{\epsilon_{n}, \eta}-\hat{\mathcal{L}}_{\mathrm{P} \mid \mathrm{SX}} \psi^{\epsilon_{n}, \eta}\right\}\left(\tilde{\zeta}^{\epsilon_{n}}, \tilde{x}^{\epsilon_{n}}\right) \leq 0 \quad \text { for all } n \geq 1 .
$$

Moreover, (5.27), (5.18) and Lemma 3.4 imply that we can apply Lemma 5.4 to $\psi^{\epsilon_{n}, \eta}$. For $n$ large enough:

$0 \geq\left(-\frac{\pi_{p p}}{2\left(\pi_{p}\right)^{2}} \sigma^{2} \xi_{\epsilon_{n}}^{2}-\mathcal{H} \bar{\varphi}^{\epsilon_{n}}-\mathcal{L}_{\mathrm{x} \mid \mathrm{SP}}^{\hat{a}} \phi^{\epsilon_{n}}-\frac{\sigma^{2} \delta^{2}(1+\eta)\left(\varpi_{\xi \xi} \circ \xi_{\epsilon_{n}}\right)}{2}+R_{\epsilon_{n}}\right)\left(\tilde{\zeta}^{\epsilon_{n}}, \tilde{x}^{\epsilon_{n}}\right)$,

where

$$
\bar{\varphi}^{\epsilon_{n}}:=\Delta_{\epsilon_{n}}+\varphi+\phi^{\epsilon_{n}}
$$

and $R_{\epsilon_{n}}\left(\tilde{\zeta}^{\epsilon_{n}}, \tilde{x}^{\epsilon_{n}}\right) \rightarrow 0$ as $n \rightarrow \infty$. Sending $n \rightarrow \infty$ and using (15.22), (15.28) and (5.29) provides (5.26).

Step 5: We can now conclude the proof. By the construction of $\varpi$ as a solution of the first corrector equation (3.7) and by (5.30), we have

$$
\frac{1}{2}\left(\frac{\pi_{p p}}{\left(\pi_{p}\right)^{2}} \sigma^{2}\right)\left(\zeta_{o}\right) \bar{\xi}^{2}+\frac{1}{2}\left(\sigma^{2} \delta^{2}\right)\left(\zeta_{o}\right) \varpi_{\xi \xi}\left(\zeta_{o}, \bar{\xi}\right)=h\left(\zeta_{o}\right)
$$


which plugged into (5.26) gives

$$
-\mathcal{H} \varphi\left(\zeta_{o}\right) \leq h\left(\zeta_{o}\right)+\eta \frac{1}{2} \sigma^{2} \delta\left(\zeta_{o}\right)^{2} \varpi_{\xi \xi}\left(\zeta_{o}, \bar{\xi}\right) .
$$

Finally we note that, although $\bar{\xi}$ as constructed in Step 4 above depends on $\eta, \zeta_{o}$ does not depend on this parameter, and therefore $\left|\varpi_{\xi \xi}\left(\zeta_{o}, \cdot\right)\right|$ is bounded by Lemma 3.4 Sending $\eta \rightarrow 0$ in the above inequality leads to

$$
-\mathcal{H} \varphi\left(\zeta_{o}\right) \leq h\left(\zeta_{o}\right)
$$

\subsection{Viscosity supersolution property}

For sake of completeness, we report here [47, Lemma 5.4] that will be used in the proof below.

Lemma 5.6. For all $\eta \in(0,1)$, there exists $c_{\eta}>1$ and a smooth function $\mathfrak{h}_{\eta}: \mathbb{R} \rightarrow[0,1]$ satisfying $\mathfrak{h}_{\eta}=1$ on $[-1,1], \mathfrak{h}_{\eta}=0$ on $\left[-c_{\eta}, c_{\eta}\right]^{c}$ and

$$
|x|\left|\mathfrak{h}_{\eta}^{\prime}(x)\right| \leq \eta, \quad \text { and } \quad|x|\left|\mathfrak{h}_{\eta}^{\prime \prime}(x)\right| \leq 2 C^{*},
$$

for some constant $C^{*}>0$ independent of $\eta$.

Proposition 5.7. Let the conditions of Theorem 3.7 hold. Then, $u_{*}$ is a viscosity supersolution of (3.5).

Proof. Let $\zeta_{o} \in \mathfrak{D}_{<T}$ and $\varphi \in C^{1,2}\left(\mathfrak{D}_{<T}\right)$ be such that

$$
\min _{\mathfrak{D}<T}(\text { strict })\left(u_{*}-\varphi\right)=\left(u_{*}-\varphi\right)\left(\zeta_{o}\right)=0 .
$$

By Lemma 5.3 and the continuity of $\varphi$, there exists $\left(\zeta^{\epsilon}\right)_{\epsilon>0}$ such that

$$
\begin{gathered}
\zeta^{\epsilon} \underset{\epsilon \downarrow 0}{\longrightarrow} \zeta_{o}, \quad x^{\epsilon}:=\theta\left(\zeta^{\epsilon}\right) \underset{\epsilon \downarrow 0}{\longrightarrow} \theta\left(\zeta_{o}\right)=: x_{o}, \\
u_{*}^{\epsilon}\left(\zeta^{\epsilon}, x^{\epsilon}\right) \underset{\epsilon \downarrow 0}{\longrightarrow} u_{*}\left(\zeta_{o}\right) \text { and } \Delta_{\epsilon}:=u_{*}^{\epsilon}\left(\zeta^{\epsilon}, x^{\epsilon}\right)-\varphi\left(\zeta^{\epsilon}\right) \underset{\epsilon \downarrow 0}{\longrightarrow} 0 .
\end{gathered}
$$

Let $r_{o}>0$ and $\epsilon_{o} \in(0,1]$ be such that

$$
\left|\zeta^{\epsilon}-\zeta_{o}\right| \leq \frac{r_{o}}{2} \quad \text { and } \quad\left|\Delta_{\epsilon}\right| \leq 1 \quad \text { for all } \epsilon \leq \epsilon_{0} .
$$

Step 1: We fix $\epsilon \in\left(0, \epsilon_{o}\right]$ and construct a first test function for $u_{*}^{\epsilon}$.

Since $\varphi$ is smooth, there exists a constant $M<\infty$ such that

$$
\sup \left\{\varphi(\zeta) ; \zeta \in \bar{B}_{r_{o}}\left(\zeta_{o}\right)\right\} \leq M-4 .
$$

By (5.35), there exists a finite $d>0$ such that $\left|\zeta-\zeta^{\epsilon}\right|^{4} \geq d$ for all $\zeta \in \partial B_{r_{o}}\left(\zeta_{o}\right)$. We fix $c_{o}>0$ such that $c_{o} d \geq M$ and define

$$
\phi^{\epsilon}(\zeta):=\varphi(\zeta)+\Delta_{\epsilon}-c_{o}\left(\left|\zeta-\zeta^{\epsilon}\right|^{4}\right) .
$$

It follows from (5.35), (5.36) and the choice of $c_{o}$ that

$$
-\phi^{\epsilon} \geq 3 \quad \text { on } \quad \partial B_{r_{o}}\left(\zeta_{o}\right) .
$$

Observe for later use that

$$
\left(u_{*}^{\epsilon}-\phi^{\epsilon}\right)\left(\zeta^{\epsilon}, x^{\epsilon}\right)=0
$$


by the definition of $\Delta_{\epsilon}$.

For $\eta \in(0,1)$, we now set

$$
\psi^{\epsilon, \eta}:=v+\epsilon^{2} \phi^{\epsilon}+\epsilon^{4}(1-\eta)\left(\varpi H_{\eta}\right) \circ \xi_{\epsilon},
$$

in which

$$
H_{\eta}: \xi \in \mathbb{R} \mapsto \mathfrak{h}_{\eta}\left(\frac{\xi}{\xi_{*}}\right),
$$

for some $\xi_{*} \geq 1$ to be chosen later on, see (5.49) in Step 6, and where $\mathfrak{h}_{\eta}$ is as in Lemma 5.6 .

Step 2: Let $\mathcal{Q}_{o}:=\bar{B}_{r_{o}}\left(\zeta_{o}\right) \times \mathbb{R}$ and fix $\epsilon \in\left(0, \epsilon_{o}\right]$. We now show that, for each $n \geq 1$, there exists $\left(\hat{\zeta}^{\epsilon, n}, \hat{x}^{\epsilon, n}\right) \in \operatorname{Int}\left(\mathcal{Q}_{o}\right)$ satisfying

$$
I^{\epsilon, \eta}\left(\hat{\zeta}^{\epsilon, n}, \hat{x}^{\epsilon, n}\right) \leq \inf _{\mathcal{Q}_{o}} I^{\epsilon, \eta}+\frac{1}{2 n},
$$

in which

$$
I^{\epsilon, \eta}:=\epsilon^{-2}\left(v_{*}^{\epsilon}-\psi^{\epsilon, \eta}\right)=u_{*}^{\epsilon}-\phi^{\epsilon}-\epsilon^{2}(1-\eta)\left(\varpi H_{\eta}\right) \circ \xi_{\epsilon} .
$$

Note that $\xi_{\epsilon}\left(\zeta^{\epsilon}, x^{\epsilon}\right)=0$ since $x^{\epsilon}=\theta\left(\zeta^{\epsilon}\right)$. Recalling that $\varpi(\cdot, 0)=0$ by Lemma 3.4 (5.38) implies that

$$
I^{\epsilon, \eta}\left(\zeta^{\epsilon}, x^{\epsilon}\right)=0 .
$$

On the other hand, (5.40) combined with Remark 5.2, Remark 3.5 and Lemma 5.6 implies that

$$
I^{\epsilon, \eta} \geq-\phi^{\epsilon}-\epsilon^{2}(1-\eta)\left\{\left|\xi_{\epsilon}\right| \mathbf{1}_{\left\{\left|\xi_{\epsilon}\right| \leq c_{\eta} \xi_{*}\right\}}\right\} \geq-\phi^{\epsilon}-\epsilon^{2}(1-\eta) c_{\eta} \xi_{*} .
$$

In particular,

$$
I^{\epsilon, \eta} \geq-\phi^{\epsilon}-1 \quad \text { if } \epsilon \leq \epsilon_{\eta}:=\epsilon_{o} \wedge\left((1-\eta) c_{\eta} \xi_{*}\right)^{-\frac{1}{2}} .
$$

The set $\bar{B}_{r_{o}}\left(\zeta_{o}\right)$ being compact, the inf over $\bar{B}_{r_{o}}\left(\zeta_{o}\right)$ of the right-hand side is finite, which proves our claim.

Step 3: For $\eta \in(0,1), \epsilon \in\left(0, \epsilon_{\eta}\right]$ and $n \geq 1$, we now construct a $C^{2}$ function $\psi^{\epsilon, \eta, n}$ and $\left(\zeta^{\epsilon, n}, x^{\epsilon, n}\right) \in \operatorname{Int}\left(\mathcal{Q}_{o}\right)$ such that

$$
\min _{\mathcal{Q}_{o}}\left(v^{\epsilon}-\psi^{\epsilon, \eta, n}\right)=\left(v^{\epsilon}-\psi^{\epsilon, \eta, n}\right)\left(\zeta^{\epsilon, n}, x^{\epsilon, n}\right) .
$$

Let $f \in C_{b}^{\infty}(\mathbb{R})$ be an even function satisfying $0 \leq f \leq 1, f(0)=1$ and $f(x)=0$ whenever $|x| \geq 1$. We set

$$
\psi^{\epsilon, \eta, n}(\cdot, x):=\psi^{\epsilon, \eta}(\cdot, x)+\frac{\epsilon^{2}}{n} f\left(x-\hat{x}^{\epsilon, n}\right)
$$

and

$$
I^{\epsilon, \eta, n}(\cdot, x):=\frac{1}{\epsilon^{2}}\left(v_{*}^{\epsilon}-\psi^{\epsilon, \eta, n}\right)(\cdot, x)=I^{\epsilon, \eta}(\cdot, x)-\frac{1}{n} f\left(x-\hat{x}^{\epsilon, n}\right) .
$$

By (5.39) and the identity $f(0)=1$,

$$
I^{\epsilon, \eta, n}\left(\hat{\zeta}^{\epsilon, n}, \hat{x}^{\epsilon, n}\right)=I^{\epsilon, \eta}\left(\hat{\zeta}^{\epsilon, n}, \hat{x}^{\epsilon, n}\right)-\frac{1}{n} \leq \inf _{\mathcal{Q}_{o}} I^{\epsilon, \eta}-\frac{1}{2 n} .
$$

Moreover, by the definition of $f$,

$$
I^{\epsilon, \eta, n}=I^{\epsilon, \eta} \text { on } \mathcal{Q}_{o} \backslash \mathcal{Q}_{1}^{n} \text {, where } \mathcal{Q}_{1}^{n}:=\left\{(\zeta, x) \in \mathcal{Q}_{o} \text { s.t. }\left|x-\hat{x}^{\epsilon, n}\right| \leq 1\right\} .
$$


Since $\left(\hat{\zeta}^{\epsilon, n}, \hat{x}^{\epsilon, n}\right) \in \mathcal{Q}_{1}^{n}$, the later combined with (5.43) implies that

$$
\inf _{\mathcal{Q}_{1}^{n}} I^{\epsilon, \eta, n}<\inf _{\mathcal{Q}_{o}} I^{\epsilon, \eta} \leq \inf _{\mathcal{Q}_{o} \backslash \mathcal{Q}_{1}^{n}} I^{\epsilon, \eta}=\inf _{\mathcal{Q}_{o} \backslash \mathcal{Q}_{1}^{n}} I^{\epsilon, \eta, n}
$$

so that

$$
\inf _{\mathcal{Q}_{o}} I^{\epsilon, \eta, n}=\inf _{\mathcal{Q}_{1}^{n}} I^{\epsilon, \eta, n} .
$$

By the lower semi-continuity of $I^{\epsilon, \eta, n}$ and the compactness of $\mathcal{Q}_{1}^{n}$, we can then find $\left(\zeta^{\epsilon, n}, x^{\epsilon, n}\right) \in \mathcal{Q}_{o}$ which minimizes $I^{\epsilon, \eta, n}$ on $\mathcal{Q}_{o}$. It remains to show that it belongs to $\operatorname{Int}\left(\mathcal{Q}_{o}\right)$. Indeed, the left hand-side of (5.35), the property $f \geq 0$, and (5.41) imply that

$$
I^{\epsilon, \eta, n}\left(\zeta^{\epsilon, n}, x^{\epsilon, n}\right) \leq I^{\epsilon, \eta, n}\left(\zeta^{\epsilon}, x^{\epsilon}\right) \leq I^{\epsilon, \eta}\left(\zeta^{\epsilon}, x^{\epsilon}\right)=0,
$$

whereas by (5.37), (5.42) and the fact that $-f \geq-1$, we have

$$
I^{\epsilon, \eta, n} \geq I^{\epsilon, \eta}-\frac{1}{n} \geq 2-\frac{1}{n}>0 \quad \text { on } \partial \mathcal{Q}_{o}=\partial B_{r_{o}}\left(\zeta_{o}\right) \times \mathbb{R} .
$$

Step 4: Given $\eta \in(0,1)$ and $\epsilon \in\left(0, \epsilon_{\eta}\right]$, we now show that there exists $N_{\epsilon, \eta} \geq 1$ such that

$$
-\left(\mathcal{L}_{S X} v^{\epsilon}+\hat{\mathcal{L}}_{P \mid S X}\right) \psi^{\epsilon, \eta, n}\left(\zeta^{\epsilon, n}, x^{\epsilon, n}\right) \geq 0 \text { for } n \geq N_{\epsilon, \eta} .
$$

In view of step 3 and Theorem 2.1, it suffices to show that

$$
\max \left\{-\epsilon^{3}+1+\psi_{x}^{\epsilon, \eta, n} ;-\epsilon^{3}-\left(1+\psi_{x}^{\epsilon, \eta, n}\right)\right\}\left(\zeta^{\epsilon, n}, x^{\epsilon, n}\right)<0,
$$

or equivalently that

$$
\left|1+\psi_{x}^{\epsilon, \eta, n}\right|\left(\zeta^{\epsilon, n}, x^{\epsilon, n}\right)<\epsilon^{3} .
$$

Recalling that $f \in C_{b}^{\infty}(\mathbb{R})$ is even, we first compute

$$
1+\psi_{x}^{\epsilon, \eta, n}\left(\zeta^{\epsilon, n}, x^{\epsilon, n}\right)=\epsilon^{3}(1-\eta)\left(\varpi H_{\eta}\right)_{\xi} \circ \xi_{\epsilon}\left(\zeta^{\epsilon, n}, x^{\epsilon, n}\right)+\frac{\epsilon^{2}}{n} f^{\prime}\left(\left|x^{\epsilon, n}-\hat{x}^{\epsilon, n}\right|\right) .
$$

Since $f \in C_{b}^{\infty}(\mathbb{R})$ is constant outside $[-1,1]$, there exists $0<c_{f}<+\infty$, which does not depend on $\epsilon$ nor $n$, such that

$$
\left|1+\psi_{x}^{\epsilon, \eta, n}\left(\zeta^{\epsilon, n}, x^{\epsilon, n}\right)\right|=\epsilon^{3}(1-\eta)\left(\left|\varpi_{\xi} H_{\eta}\right|+\left|\varpi H_{\eta}^{\prime}\right|\right) \circ \xi_{\epsilon}\left(\zeta^{\epsilon, n}, x^{\epsilon, n}\right)+\frac{\epsilon^{2} c_{f}}{n} .
$$

In view of (3.7), (ii) of Lemma 3.4, Remark 3.5 and the fact that $\left|H_{\eta}\right| \leq 1$ by Lemma [5.6. this implies that

$$
\left|1+\psi_{x}^{\epsilon, \eta, n}\left(\zeta^{\epsilon, n}, x^{\epsilon, n}\right)\right|=\epsilon^{3}(1-\eta)\left(1+\frac{\left|\xi_{\epsilon}\right|}{\xi_{*}}\left|\mathfrak{h}_{\eta}^{\prime}\right|\left(\frac{\xi_{\epsilon}}{\xi_{*}}\right)\right)\left(\zeta^{\epsilon, n}, x^{\epsilon, n}\right)+\frac{\epsilon^{2} c_{f}}{n} .
$$

Recalling from Lemma [5.6 that $|x|\left|\mathfrak{h}_{\eta}^{\prime}(x)\right| \leq \eta$ for $x \in \mathbb{R}$, we finally obtain

$$
\left|1+\psi_{x}^{\epsilon, \eta, n}\left(\zeta^{\epsilon, n}, x^{\epsilon, n}\right)\right| \leq \epsilon^{3}\left(1-\eta^{2}\right)+\frac{\epsilon^{2} c_{f}}{n}<\epsilon^{3} \quad \text { for all } n \geq 1+\frac{c_{f}}{\epsilon \eta^{2}}=: N_{\epsilon, \eta} .
$$

Step 5: We now show that $\left\{\xi_{\epsilon}\left(\zeta^{\epsilon, n}, x^{\epsilon, n}\right) ; \epsilon \in\left(0, \epsilon_{\eta}\right], n \geq N_{\epsilon, \eta}\right\}$ is uniformly bounded.

We first appeal to Lemma [5.4, recall Assumption 3.3. Lemma 3.4 and that $\left(\zeta^{\epsilon, n}\right.$, $\left.n \geq 1, \epsilon \in\left(0, \epsilon_{\eta}\right]\right)$ is bounded, see step 3 . Since $\phi^{\epsilon}$ does not depend on the $x$-variable, this implies

$$
-\epsilon^{-2}\left(\mathcal{L}_{\mathrm{SX}}+\hat{\mathcal{L}}_{\mathrm{P} \mid \mathrm{SX}}\right) \psi^{\epsilon, \eta, n}=-\frac{1}{2} \frac{\pi_{p p}}{\left(\pi_{p}\right)^{2}} \sigma^{2} \xi_{\epsilon}^{2}-\mathcal{H} \phi^{\epsilon}-\frac{1-\eta}{2} \sigma^{2} \delta^{2}(\varpi H)_{\xi \xi} \circ \xi_{\epsilon}+R^{\epsilon, n},
$$


at the point $\left(\zeta^{\epsilon, n}, x^{\epsilon, n}\right)$, in which, by (ii) of Lemma 5.4

$$
\left|R^{\epsilon, n}\right| \leq C_{\eta}\left(1+\epsilon\left|\xi_{\epsilon}\right|+\epsilon^{2}\left|\xi_{\epsilon}\right|^{2}\right)\left(\zeta^{\epsilon, n}, x^{\epsilon, n}\right),
$$

for some $C_{\eta}>0$ independent on $n$ and $\epsilon$. By (5.45), we then have

$$
\left(\frac{1}{2} \frac{\pi_{p p}}{\left(\pi_{p}\right)^{2}} \sigma^{2} \xi_{\epsilon}^{2}\right)\left(\zeta^{\epsilon, n}, x^{\epsilon, n}\right)-\left|R^{\epsilon, n}\right| \leq-\left(\mathcal{H} \phi^{\epsilon}+\frac{1-\eta}{2} \sigma^{2} \delta^{2}(\varpi H)_{\xi \xi} \circ \xi_{\epsilon}\right)\left(\zeta^{\epsilon, n}, x^{\epsilon, n}\right) .
$$

We first consider the last term of the previous inequality. By Lemma 3.4 and the boundedness of $\left(\zeta^{\epsilon, n}, \epsilon \in\left(0, \epsilon_{\eta}\right], n \geq 1\right)$, we can find $C_{\eta}>0$, independent on $n, \epsilon$ and $\eta$, such that such that $\left|\varpi_{\xi \xi} \circ \xi_{\epsilon}\right|\left(\zeta^{\epsilon, n}, x^{\epsilon, n}\right) \leq C$. The same Lemma and Remark 3.5 also imply that $\left|\varpi_{\xi} \circ \xi_{\epsilon}\right|\left(\zeta^{\epsilon, n}, x^{\epsilon, n}\right) \leq 1$ and $\left|\varpi \circ \xi_{\epsilon}\right|\left(\zeta^{\epsilon, n}, x^{\epsilon, n}\right) \leq\left|\xi_{\epsilon}\left(\zeta^{\epsilon, n}, x^{\epsilon, n}\right)\right|$. Using Lemma 5.6] and the fact that $\xi_{*} \geq 1$ and $\eta \leq 1$, it follows that, at the point $\left(\zeta^{\epsilon, n}, x^{\epsilon, n}\right)$,

$$
\begin{aligned}
\left|\left(\varpi H_{\eta}\right)_{\xi \xi}\right| \circ \xi_{\epsilon} & =\left|\varpi_{\xi \xi} H_{\eta}+2 \varpi_{\xi} H_{\eta}^{\prime}+\varpi H_{\eta}^{\prime \prime}\right| \circ \xi_{\epsilon} \\
& \leq C_{\eta}+\frac{2}{\xi_{*}}\left|\mathfrak{h}_{\eta}^{\prime}\left(\frac{\xi_{\epsilon}}{\xi_{*}}\right)\right| \mathbf{1}_{\left[\xi_{*}, c_{\eta} \xi_{*}\right]}\left(\left|\xi_{\epsilon}\right|\right)+\frac{\left|\xi_{\epsilon}\right|}{\left(\xi_{*}\right)^{2}}\left|\mathfrak{h}_{\eta}^{\prime \prime}\left(\frac{\xi_{\epsilon}}{\xi_{*}}\right)\right| \\
& \leq C_{\eta}+\frac{2\left|\xi_{\epsilon}\right|}{\left(\xi_{*}\right)^{2}}\left|\mathfrak{h}_{\eta}^{\prime}\left(\frac{\xi_{\epsilon}}{\xi_{*}}\right)\right|+\frac{\left|\xi_{\epsilon}\right|}{\left(\xi_{*}\right)^{2}}\left|\mathfrak{h}_{\eta}^{\prime \prime}\left(\frac{\xi_{\epsilon}}{\xi_{*}}\right)\right| \\
& \leq C_{\eta}+\frac{2}{\xi_{*}}\left(\eta+C^{*}\right) \\
& \leq C_{\eta}+2\left(1+C^{*}\right)=: \bar{C}_{\eta} .
\end{aligned}
$$

Plugging this result into (5.48) leads to

$$
\left(\frac{1}{2} \frac{\pi_{p p}}{\left(\pi_{p}\right)^{2}} \sigma^{2} \xi_{\epsilon}^{2}\right)\left(\zeta^{\epsilon, n}, x^{\epsilon, n}\right)-\left|R^{\epsilon, n}\right| \leq-\left(\mathcal{H} \phi^{\epsilon}-\bar{C} \frac{1-\eta}{2} \sigma^{2} \delta^{2}\right)\left(\zeta^{\epsilon, n}, x^{\epsilon, n}\right) .
$$

The later combined with Assumption 3.3, (2.2), (5.47) and the fact that both $\zeta^{\epsilon, n}$ and $\zeta^{\epsilon}$ lie in $B_{r_{o}}\left(\zeta_{o}\right)$, and the identity $\epsilon \xi_{\epsilon}\left(\zeta^{\epsilon, n}, x^{\epsilon, n}\right)=x^{\epsilon, n}-\theta\left(\zeta^{\epsilon, n}\right)$, allows us to find a constants $K_{\eta}>0$, independent on $n$ and $\epsilon$, such that

$$
\left[\left(\xi_{\epsilon}\right)^{2}-K_{\eta}\left(1+\left|\epsilon \xi_{\epsilon}\right|+\left|\epsilon \xi_{\epsilon}\right|^{2}\right)\right]\left(\zeta^{\epsilon, n}, x^{\epsilon, n}\right) \leq 0 .
$$

This proves our claim.

Step 6: We are now in position to conclude the proof.

By the previous step, for all $\epsilon \in\left(0, \epsilon_{\eta}\right]$, we may assume, after possibly passing to a subsequence, that $\left(\zeta^{\epsilon, n}, x^{\epsilon, n}, \xi_{\epsilon}\left(\zeta^{\epsilon, n}, x^{\epsilon, n}\right)\right) \rightarrow\left(\bar{\zeta}^{\epsilon}, \theta\left(\bar{\zeta}^{\epsilon}\right), \bar{\xi}^{\epsilon}\right) \in \mathfrak{D} \times \mathbb{R}^{2}$ as $n \rightarrow \infty$. Classical arguments then show that $\left(\bar{\zeta}^{\epsilon}, \bar{\xi}^{\epsilon}\right) \rightarrow\left(\zeta_{o}, \hat{\xi}\right)$ for some bounded $\hat{\xi} \in \mathbb{R}$, and therefore $\theta\left(\bar{\zeta}^{\epsilon}\right) \rightarrow \theta\left(\zeta_{o}\right)=x_{o}$, as $\epsilon \rightarrow 0$, after possibly passing to a subsequence. Moreover, (i) of Lemma 5.4 now implies that $R^{\epsilon, n} \rightarrow 0$ as $n \rightarrow \infty$ and then $\epsilon \rightarrow 0$. Hence, sending $n \rightarrow \infty$ and then $\epsilon \rightarrow 0$ in (5.48) provides

$$
\frac{1}{2}\left(\frac{\pi_{p p}}{\left(\pi_{p}\right)^{2}} \sigma^{2}\right)\left(\zeta_{o}\right) \hat{\xi}^{2} \leq-\mathcal{H} \varphi\left(\zeta_{o}\right)-\frac{1-\eta}{2}\left\{\sigma^{2} \delta^{2}\left(\varpi H_{\eta}\right)_{\xi \xi}\right\}\left(\zeta_{o}, \hat{\xi}\right) .
$$

The same arguments as in step 5 then shows that

$$
\hat{\xi}^{2} \leq\left(\frac{-\mathcal{H} \varphi+\sigma^{2} \delta^{2} \bar{C}(1-\eta) / 2}{\frac{1}{2} \frac{\pi_{p p}}{\left(\pi_{p}\right)^{2}} \sigma^{2}}\right)\left(\zeta_{o}\right) .
$$


We now choose $\xi_{*} \geq 1$ defined by

$$
\left(\xi_{*}\right)^{2}:=2 \vee 2\left(\frac{-\mathcal{H} \varphi+\sigma^{2} \delta^{2} \bar{C} / 2}{\frac{1}{2} \frac{\pi_{p p}}{\left(\pi_{p}\right)^{2}} \sigma^{2}}\right)\left(\zeta_{o}\right) .
$$

Note that all the quantities on the right-hand side are given a-priori. Then, $|\hat{\xi}|<\xi_{*}$. In particular, $H_{\eta}=1$ in a neighborhood of $\hat{\xi}$, see Lemma [5.6, and the above then implies that

$$
\frac{1}{2}\left(\frac{\pi_{p p}}{\left(\pi_{p}\right)^{2}} \sigma^{2}\right)\left(\zeta_{o}\right) \hat{\xi}^{2} \leq-\mathcal{H} \varphi\left(\zeta_{o}\right)-\frac{1-\eta}{2}\left(\sigma^{2} \delta^{2} \varpi_{\xi \xi}\right)\left(\zeta_{o}, \hat{\xi}\right) .
$$

Since $\varpi$ is solution of (3.7), it follows that

$$
\mathcal{H} \varphi\left(\zeta_{o}\right) \leq-h\left(\zeta_{o}\right)+\frac{\eta}{2} \sigma^{2} \delta^{2} \varpi_{\xi \xi}\left(\zeta_{o}, \hat{\xi}\right) .
$$

It remains to let $\eta \rightarrow 0$ and recall from Lemma 3.4 that $\left|\varpi_{\xi \xi}\left(\zeta_{o}, \cdot\right)\right|$ is bounded.

\subsection{The Terminal condition}

Proposition 5.8. Let the conditions of Theorem 3.7 hold. Then, $u^{*}=u_{*}=0$ on $\mathfrak{D}_{T}$.

Proof. The fact that $u_{*}(T, \cdot) \geq 0$ follows from Remark 5.2 In the following, we prove that $u^{*}(T, \cdot) \leq 0$. We assume to the contrary that we can find $\left(T, s_{o}, p_{o}\right):=\zeta_{o} \in \mathfrak{D}_{T}$ such that

$$
u^{*}\left(\zeta_{o}\right) \geq 4 \kappa \quad \text { for some } \quad \kappa>0
$$

and work towards a contradiction.

Step 1: We construct a test function $\psi^{\epsilon}$ for $v^{\epsilon *}$ and show that $v^{\epsilon *}-\psi^{\epsilon}$ admits a local maximizer $\left(\tilde{t}_{\epsilon}, \tilde{s}_{\epsilon}, \tilde{p}_{\epsilon}, \tilde{x}_{\epsilon}\right)=\left(\tilde{\zeta}_{\epsilon}, \tilde{x}_{\epsilon}\right) \in \mathfrak{D}_{<T} \times \mathbb{R}$.

By Lemma 5.3 there are $\left(\zeta_{\epsilon}\right)_{\epsilon>0} \subset \mathfrak{D}$ and $x_{o} \in \mathbb{R}$ such that

$$
\zeta_{\epsilon} \underset{\epsilon \downarrow 0}{\longrightarrow} \zeta_{o}, \quad x_{\epsilon}:=\theta\left(\zeta_{\epsilon}\right) \underset{\epsilon \downarrow 0}{\longrightarrow} \theta\left(\zeta_{o}\right)=: x_{o} \quad \text { and } \quad u^{\epsilon *}\left(\zeta_{\epsilon}, x_{\epsilon}\right) \underset{\epsilon \downarrow 0}{\longrightarrow} u^{*}\left(\zeta_{o}\right),
$$

in which $\left(t_{\epsilon}, s_{\epsilon}, p_{\epsilon}\right):=\zeta_{\epsilon}$. Note that, after possibly passing to a subsequence, one can assume that

$$
\zeta_{\epsilon} \in \mathfrak{D}_{<T} \text { for all } \epsilon>0
$$

Indeed, Theorem 2.1 and Theorem 2.3 imply that

$$
u^{\epsilon *}(\zeta, x) \leq \epsilon|x| \text { for all }(\zeta, x) \in \mathfrak{D}_{T} \times \mathbb{R},
$$

which would lead to a contradiction of (5.50) if (5.52) was not satisfied, at least along a subsequence, since, by (5.51), $\left(\zeta_{\epsilon}, x_{\epsilon}\right)_{\epsilon>0}$ is bounded.

Combining arguments similar to those of the proof of Proposition 5.5 (Step 1) with (5.50), (5.51), Assumptions 3.2 and 3.3 allow us to construct $0<r_{o} \leq \bar{r}_{o}, \epsilon_{o} \in(0,1], c_{o}>$ 0 and $\iota>0$ such that, for all $\epsilon \in\left(0, \epsilon_{o}\right]$,

$$
\begin{gathered}
\left(\zeta_{\epsilon}, x_{\epsilon}\right) \in B_{o, \frac{1}{2}} \quad \text { and } \quad u^{\epsilon *}\left(\zeta_{\epsilon}, x_{\epsilon}\right) \geq 2 \kappa, \\
\pi_{p} \geq 2 \iota \quad \text { on } B_{o}, \\
u^{\epsilon *}-\bar{\phi}\left(\cdot ; s_{\epsilon}, p_{\epsilon}\right)<0 \quad \text { on } \quad B_{o} \backslash B_{o, \frac{1}{2}}
\end{gathered}
$$


where $B_{o}:=\left[T-r_{o}, T\right] \times \bar{B}_{r_{o}}\left(s_{o}, p_{o}\right) \times \bar{B}_{\bar{r}_{o}}\left(x_{o}\right)$,

$$
\begin{gathered}
B_{o, \frac{1}{2}}:=\left\{(\zeta, x) \in B_{o} \text { s.t. } \zeta \in\left[T-\frac{r_{o}}{2}, T\right] \times \bar{B}_{\frac{r_{o}}{2}}\left(s_{o}, p_{o}\right) \text { and } x \in \bar{B}_{\frac{\bar{r}_{o}}{2}}\left(x_{o}\right)\right\}, \\
\bar{\phi}\left(\cdot ; s_{\epsilon}, p_{\epsilon}\right):(t, s, p, x) \in \mathfrak{D} \times \mathbb{R} \longmapsto c_{o}\left(\left|s_{\epsilon}-s\right|^{4}+\left|p_{\epsilon}-p\right|^{4}+|x-\theta(t, s, p)|^{2}\right) .
\end{gathered}
$$

Recalling (5.52) and Assumption 3.3, we may then define, for each $\epsilon \in\left(0, \epsilon_{o}\right]$, the smooth function $\psi^{\epsilon}:=v+\epsilon^{2} \phi^{\epsilon}$ with

$$
\phi^{\epsilon}:(t, s, p, x) \in \mathfrak{D} \times \mathbb{R} \longmapsto \kappa \frac{T-t}{T-t_{\epsilon}}+\bar{\phi}\left(t, s, p, x ; s_{\epsilon}, p_{\epsilon}\right) .
$$

By the upper semi-continuity of $v^{\epsilon *}$, we deduce from (5.53) and (5.55) that $v^{\epsilon *}-\psi^{\epsilon}$ admits on $B_{o}$ a local maximizer $\left(\tilde{\zeta}_{\epsilon}, \tilde{x}_{\epsilon}\right) \in B_{o, \frac{1}{2}}$ for every $\epsilon \in\left(0, \epsilon_{o}\right]$, and that moreover

$$
u^{\epsilon *}\left(\tilde{\zeta}_{\epsilon}, \tilde{x}_{\epsilon}\right) \geq \kappa .
$$

By the argument used above, this implies that $\tilde{\zeta}_{\epsilon} \in \mathfrak{D}_{<T}$ for all $\epsilon \in\left(0, \epsilon_{o}\right]$ after possibly choosing a subsequence.

Step 2: We now show that $\left(\xi_{\epsilon}\left(\tilde{\zeta}_{\epsilon}, \tilde{x}_{\epsilon}\right)\right)_{\epsilon \in\left(0, \epsilon_{o}\right]}$ is uniformly bounded.

We fix $\epsilon \in\left(0, \epsilon_{o}\right]$. The previous step and Theorem 2.1 imply that

$$
\max \left\{-\left(\mathcal{L}_{\mathrm{SX}}+\hat{\mathcal{L}}_{\mathrm{P} \mid \mathrm{SX}}\right) \psi^{\epsilon} ;-\epsilon^{3}+1+\psi_{x}^{\epsilon} ;-\epsilon^{3}-1-\psi_{x}^{\epsilon}\right\}\left(\tilde{\zeta}_{\epsilon}, \tilde{x}_{\epsilon}\right) \leq 0 .
$$

Straightforward computations based on the gradient constraints give

$$
-\frac{1}{2 c_{o}} \leq \xi_{\epsilon}\left(\tilde{\zeta}_{\epsilon}, \tilde{x}_{\epsilon}\right) \leq \frac{1}{2 c_{o}} .
$$

Step 3: We can now conclude the proof.

We fix $\epsilon \in\left(0, \epsilon_{o}\right]$ and focus on the second order operator in (5.56). It follows from (5.54) that $\psi_{p}^{\epsilon}\left(\tilde{\zeta}_{\epsilon}, \tilde{x}_{\epsilon}\right) \geq \iota>0$, after possibly changing $\epsilon_{o}$. Hence, Step 2 and (i) of Lemma 5.4 imply that

$$
\left(-\frac{1}{2} \frac{\pi_{p p}}{\left(\pi_{p}\right)^{2}} \sigma^{2} \xi_{\epsilon}^{2}-\mathcal{H} \phi^{\epsilon}-\mathcal{L}_{\mathrm{X} \mid \mathrm{SP}}^{\hat{a}} \phi^{\epsilon}+R^{\epsilon}\right)\left(\tilde{\zeta}_{\epsilon}, \tilde{x}_{\epsilon}\right) \leq 0
$$

where $\sup _{\epsilon \in\left(0, \epsilon_{o}\right]}\left|R^{\epsilon}\right|\left(\tilde{\zeta}_{\epsilon}, \tilde{x}_{\epsilon}\right)<\infty$. Recalling (5.57), the fact that $\left(\tilde{\zeta}_{\epsilon}, \epsilon \in\left(0, \epsilon_{o}\right]\right)$ is bounded, that $\tilde{x}^{\epsilon}=\epsilon \xi_{\epsilon}\left(\zeta_{\epsilon}\right)+\theta\left(\zeta_{\epsilon}\right)$, and Assumption 3.3, we finally deduce that

$\frac{\kappa}{T-t_{\epsilon}} \leq\left(\frac{1}{2} \frac{\pi_{p p}}{\left(\pi_{p}\right)^{2}} \sigma^{2} \frac{1}{4 c_{o}^{2}}+\left(\mathcal{H}+\mathcal{L}_{\mathrm{x} \mid \mathrm{SP}}^{\hat{a}}\right) \bar{\phi}\left(\cdot ; s_{\epsilon}, p_{\epsilon}\right)+R^{\epsilon}\right)\left(\tilde{\zeta}_{\epsilon}, \tilde{x}_{\epsilon}\right) \leq C$ for all $\epsilon \in\left(0, \bar{\epsilon}_{o}\right]$, for some constant $C>0$ (independent of $\epsilon$ ). As $t_{\epsilon} \rightarrow T$, we obtain a contradiction.

\section{Explicit resolution of the first corrector equation}

In this section, we prove Lemma 3.4. We follow the steps of [53. Namely, we look for a solution of the first order equation (3.7) with an additional condition at the boundary $\xi=0$. We fix $\zeta \in \mathfrak{D}$ and simply write $\varpi(\xi)$ for $\varpi(\zeta, \xi)$. We recall that we work under Assumption 3.3

It is natural to search for a solution of the form

$$
\varpi(\xi)= \begin{cases}k_{4} \xi^{4}+k_{2} \xi^{2}+k_{1} \xi & \xi_{1} \leq \xi \leq \xi_{0}, \\ -\xi+k_{3} & \xi \leq \xi_{1}, \\ \xi+k_{0} & \xi \geq \xi_{0},\end{cases}
$$


for some real numbers $k_{4}, k_{3}, k_{2}, k_{1}, k_{0}$ and $\xi_{1} \leq \xi_{0}$. Since the fourth order polynomial solves the second order equation, we find

$$
k_{4}=-\frac{1}{12} \frac{\pi_{p p}}{\delta^{2}\left(\pi_{p}\right)^{2}} \quad \text { and } \quad k_{2}=\frac{h}{\sigma^{2} \delta^{2}} .
$$

If we now assume that $\varpi_{\xi \xi}$ is continuous at the point $\xi_{0}$ and $\xi_{1}$, we have

$$
12 k_{4}\left(\xi_{0}\right)^{2}+2 k_{2}=12 k_{4}\left(\xi_{1}\right)^{2}+2 k_{2}=0,
$$

that is

$$
\left(\xi_{0}\right)^{2}=\left(\xi_{1}\right)^{2}=2 \frac{h}{\sigma^{2}} \times \frac{\left(\pi_{p}\right)^{2}}{\pi_{p p}},
$$

which, by the fact that $\pi_{p p}>0$, implies that $h \geq 0$ and

$$
\hat{\xi}:=\xi_{0}=-\xi_{1}=\left(2 \frac{h}{\sigma^{2}} \times \frac{\left(\pi_{p}\right)^{2}}{\pi_{p p}}\right)^{\frac{1}{2}} .
$$

Assuming now that $\varpi_{\xi}$ is continuous at the point $\xi_{0}$ and $\xi_{1}$ leads to

$$
\begin{gathered}
4 k_{4}(\hat{\xi})^{3}+2 k_{2} \hat{\xi}+k_{1}=1, \\
-4 k_{4}(\hat{\xi})^{3}-2 k_{2} \hat{\xi}+k_{1}=-1,
\end{gathered}
$$

which gives $k_{1}=0$. By substituting (6.1) into (6.2),

$$
-\frac{\pi_{p p}}{\delta^{2}\left(\pi_{p}\right)^{2}}(\hat{\xi})^{3}+\frac{6 h}{\sigma^{2} \delta^{2}} \hat{\xi}=3 .
$$

Since, by the above,

$$
h=\frac{\sigma^{2} \pi_{p p}}{2\left(\pi_{p}\right)^{2}}(\hat{\xi})^{2},
$$

we obtain

$$
\hat{\xi}=\left(\frac{3}{2} \frac{\delta^{2}\left(\pi_{p}\right)^{2}}{\pi_{p p}}\right)^{\frac{1}{3}}
$$

The remaining constants $k_{0}$ and $k_{3}$ are obtained by assuming the continuity of $\varpi$ at the points $\xi_{0}$ and $\xi_{1}$. Gathering the above terms together, we finally obtain

$$
\varpi(\xi)= \begin{cases}-\frac{1}{8 \hat{\xi}^{3}} \xi^{4}+\frac{3}{4 \hat{\xi}} \xi^{2} & -\hat{\xi} \leq \xi \leq \hat{\xi}, \\ -\xi-\frac{3 \hat{\xi}}{8} & \xi \leq-\hat{\xi}, \\ \xi-\frac{3 \hat{\xi}}{8} & \xi \geq \hat{\xi} .\end{cases}
$$

The remaining properties stated in Lemma 3.4 are straightforward under Assumption 3.3 .

\section{Verification of the assumptions in the examples}

In this section, we provide the proofs of Propositions 4.3 and 4.5. We also explain how to construct an explicit almost optimal strategy. 


\subsection{Exponential case}

We provide here the proof of Proposition 4.3 .

Proof of Proposition 4.3 First note that (4.3) together with Assumption 4.2 imply Assumption 3.3. Under the boundedness condition b. of Assumption 4.2, the function $h$ is bounded, see (4.4). It follows that the map defined in (4.7) is bounded. Moreover, standard arguments show that comparison holds in the viscosity solution sense for the above equation in the class of functions with polynomial growth, see [18. Then, Assumption 3.6 will hold if one shows that there exists $C>0$ such that

$$
0 \leq u^{\epsilon}(\zeta, x) \leq C(1+\epsilon|x|) \text { for all }(\zeta, x) \in \mathfrak{D} \times \mathbb{R} \text { and } \epsilon \in(0,1]
$$

in which the left-hand side inequality is already a consequence of Remark 5.2 This will also imply Assumption 3.2. The following arguments aim at proving the right-hand side inequality of (7.1).

Step 1. We restrict to $0<\epsilon \leq 1$. Set

$$
\psi^{\epsilon}(t, s, p, x):=v(t, s, p, x)+\epsilon^{4} \check{\varpi} \circ \xi_{\epsilon}(t, s, x) \text { for }(t, s, p, x) \in \mathfrak{D} \times \mathbb{R},
$$

in which $\check{\varpi}$ is the solution of (3.7) as constructed in Section 6 but for $\delta=\sigma=1$ and $\pi_{p}^{2} / \pi_{p p}=1$. For later use, observe that it takes non-negative values. We denote by $\check{\xi}$ the corresponding $\hat{\xi}$ and $\check{h}$ the corresponding $h$. Then, $\check{\xi}$ and $\check{h}$ are constant, and $\check{\varpi}$ depends only on $\xi$. Let us also define

$$
\begin{aligned}
\hat{a}^{\epsilon} & :=\frac{-\bar{\sigma}_{0}^{\top} D \psi^{\epsilon}}{\pi_{p}} \\
& =\eta p \sigma\left[(\theta-x)\left(1-\epsilon^{3} \varpi_{\xi} \circ \xi_{\epsilon}\right)+\epsilon^{3} \varpi_{\xi} \circ \xi_{\epsilon}\left(\frac{\lambda}{\sigma \eta}-s^{2} \bar{\pi}_{s s}\right)-\frac{\lambda}{\sigma \eta}\right]
\end{aligned}
$$

and

$$
\begin{aligned}
\mathcal{J}_{\epsilon} & :=\left\{(t, s, x) \in[0, T] \times(0, \infty) \times \mathbb{R}:-\check{\xi}(t, s)<\xi_{\epsilon}(t, s, x)<\check{\xi}(t, s)\right\} \\
& =\{(t, s, x) \in[0, T] \times(0, \infty) \times \mathbb{R}:-\epsilon \check{\xi}(t, s)<x-\theta(t, s)<\epsilon \dot{\xi}(t, s)\},
\end{aligned}
$$

recall Proposition 4.1 and (4.5). Lemma 3.4 allows one to characterize the boundaries of this domain in terms of the function $\varpi$ :

$$
\partial \mathcal{J}_{\epsilon}^{ \pm}:=\left\{\xi_{\epsilon}=\mp \check{\xi}\right\} \subset\left\{\varpi_{\xi} \circ \xi_{\epsilon}=\mp 1\right\} .
$$

For later use, note that Assumption 4.2 implies that

$$
(t, s, x) \in \mathcal{J}_{\epsilon} \Longrightarrow\left\{|x| \leq C_{K} \text { and }\left|p^{-1} \hat{a}^{\epsilon}(t, s, x, p)\right| \leq C_{K} \text { for all } p<0\right\},
$$

in which $C_{K}$ denotes from now on a generic positive constant which depends only on the constant $K>0$ of Assumption 4.2, and that may change from line to line.

We now fix $\left(t_{o}, s_{o}, x_{o}\right)$ in the closure of $\mathcal{J}_{\epsilon}$. The general case will be discussed in the last step of the proof. We define $\left(X^{\epsilon}, L^{\epsilon}\right)$ as the solution of the following Skorokhod problem

$$
\left\{\begin{array}{l}
X^{\epsilon}=x_{o}+\int_{t_{o}} X_{\tau}^{\epsilon} \frac{d S_{\tau}}{S_{\tau}}+\int_{t_{o}} d L_{\tau}^{\epsilon+}-\int_{t_{o}} d L_{\tau}^{\epsilon-}, \\
\left(\cdot, S, X^{\epsilon}\right) \in \mathcal{J}_{\epsilon} \quad d t \otimes d \mathbb{P} \text {-a.e. on }\left[t_{o}, T\right], \\
L^{\epsilon \pm}=\int_{t_{o}} \chi_{\left\{\left(\tau, S_{\tau}, X_{\tau}^{\epsilon}\right) \in \partial \mathcal{J}_{\epsilon}^{ \pm}\right\}} d L_{\tau}^{\epsilon \pm},
\end{array}\right.
$$

in which $S=S^{t_{o}, s_{o}}$ and $L^{\epsilon}=L^{\epsilon+}-L^{\epsilon-}$ where $L^{\epsilon+}, L^{\epsilon-}$ are continuous and nondecreasing. To see that the above admits a solution, first observe that Assumption 4.2 
ensures that we can find $\kappa \in \mathbb{R}$ such that $-\check{\xi}+\theta>\kappa$ on $[0, T] \times(0, \infty)$. Hence, the process $X^{\epsilon}$ satisfies the above if and only if $X^{\epsilon}-\kappa>0$, in which case

$X^{\epsilon}-\kappa=\left(x_{o}-\kappa\right) \exp \left(\int_{t_{0}}\left(\mu-\frac{1}{2} \sigma^{2}\right) d \tau+\int_{t_{0}}^{\cdot} d W_{\tau}+\int_{t_{0}}^{\cdot} d \bar{L}_{\tau}^{\epsilon+}-\int_{t_{0}}^{\cdot} d \bar{L}_{\tau}^{\epsilon-}\right) \quad$ on $\left[t_{o}, T\right]$,

with $d \bar{L}^{\epsilon \pm}=d L^{\epsilon \pm} /\left(X_{\tau}^{\epsilon}-\kappa\right)$. Thus, solving (7.6) is equivalent to finding the solution $\left(\bar{X}^{\epsilon}, \bar{L}^{\epsilon}\right)$ of the Skorohod problem

$$
\left\{\begin{array}{l}
\bar{X}^{\epsilon}=\ln \left(x_{o}-\kappa\right)+\int_{t_{0}}\left(\mu-\frac{1}{2} \sigma^{2}\right) d \tau+\int_{t_{0}} d W_{\tau}+\int_{t_{0}} d \bar{L}_{\tau}^{\epsilon+}-\int_{t_{0}} d \bar{L}_{\tau}^{\epsilon-}, \\
U^{-} \leq \bar{X}^{\epsilon} \leq U^{+} d t \otimes d \mathbb{P} \text {-a.e. on }\left[t_{o}, T\right] \\
\bar{L}^{\epsilon \pm}=\int_{t_{o}} \chi_{\left\{\bar{X}_{\tau}^{\epsilon}=U^{ \pm}\right\}} d L_{\tau}^{\epsilon \pm}
\end{array}\right.
$$

in which

$$
U^{ \pm}:=\ln (-\kappa+( \pm \epsilon \check{\xi}+\theta)(\cdot, S)) .
$$

Existence now follows from [39, Lemma 6.14], see the constructive proof for the fact that the solution is adapted.

We next define $\left(Y^{\epsilon}, P^{\epsilon}\right)$ as the solution of

$$
Y^{\epsilon}=y_{o}-\int_{t_{o}}^{\cdot}\left(1+\epsilon^{3}\right) d L_{\tau}^{\epsilon+}+\int_{t}^{\cdot}\left(1-\epsilon^{3}\right) d L_{\tau}^{\epsilon-}, P^{\epsilon}=p_{o}+\int_{t_{o}}^{\cdot} \hat{a}^{\epsilon}\left(\tau, S_{\tau}, P_{\tau}^{\epsilon}, X_{\tau}^{\epsilon}\right) d W_{\tau}
$$

in which $p_{o}<0$ and $y_{o}:=\psi^{\epsilon}\left(t_{o}, s_{o}, p_{o}, x_{o}\right)+c$ for some $c>0$ to be chosen later on. The existence of a unique strong solution to (7.7) follows from (7.5), the process $P^{\epsilon}$ is a martingale.

Step 2. We now apply Itô's Lemma to $\psi^{\epsilon}$. The definition of $\hat{a}^{\epsilon}$ and the above dynamics lead to

$$
\begin{aligned}
Y_{T}^{\epsilon}-\psi^{\epsilon}\left(T, S_{T}, P_{T}^{\epsilon}, X_{T}^{\epsilon}\right)= & c-\int_{t_{o}}^{T}\left(\mathcal{L}_{\mathrm{SX}}+\hat{\mathcal{L}}_{\mathrm{P} \mid \mathrm{Sx}}\right) \psi^{\epsilon}\left(\tau, S_{\tau}, P_{\tau}^{\epsilon}, X_{\tau}^{\epsilon}\right) d \tau \\
& -\int_{t_{o}}^{T}\left[\left(1+\epsilon^{3}\right)+\psi_{x}^{\epsilon}\left(\tau, S_{\tau}, P_{\tau}^{\epsilon}, X_{\tau}^{\epsilon}\right)\right] d L_{\tau}^{\epsilon+} \\
& +\int_{t_{o}}^{T}\left[\left(1-\epsilon^{3}\right)+\psi_{x}^{\epsilon}\left(\tau, S_{\tau}, P_{\tau}^{\epsilon}, X_{\tau}^{\epsilon}\right)\right] d L_{\tau}^{\epsilon-} \\
\geq & c-\int_{t_{o}}^{T}\left(\mathcal{L}_{\mathrm{Sx}}+\hat{\mathcal{L}}_{\mathrm{P} \mid \mathrm{Sx}}\right) \psi^{\epsilon}\left(\tau, S_{\tau}, P_{\tau}^{\epsilon}, X_{\tau}^{\epsilon}\right) d \tau \\
& -\epsilon^{3} \int_{t_{o}}^{T}\left[1+\check{\varpi}_{\xi} \circ \xi_{\epsilon}\left(\tau, S_{\tau}, X_{\tau}^{\epsilon}\right)\right] d L_{\tau}^{\epsilon+} \\
& +\epsilon^{3} \int_{t_{o}}^{T}\left[-1+\check{\varpi}_{\xi} \circ \xi_{\epsilon}\left(\tau, S_{\tau}, X_{\tau}^{\epsilon}\right)\right] d L_{\tau}^{\epsilon-} .
\end{aligned}
$$

We next appeal to (7.4) and the characterization of $L^{\epsilon+}, L^{\epsilon-}$ in (7.6) to provide a lower bound to the last expression:

$Y_{T}^{\epsilon}-\psi^{\epsilon}\left(T, S_{T}, P_{T}^{\epsilon}, X_{T}^{\epsilon}\right) \geq c-\int_{t_{o}}^{T}\left(\mathcal{L}_{\mathrm{SX}}+\hat{\mathcal{L}}_{\mathrm{P} \mid \mathrm{Sx}}\right) \psi^{\epsilon}\left(\tau, S_{\tau}, P_{\tau}^{\epsilon}, X_{\tau}^{\epsilon}\right) d \tau=: c-\epsilon^{2} E_{\epsilon}$.

We first consider the left-hand side term. The definition of $\psi^{\epsilon}$ and the identities 
$v(T, s, p, x)=g(s)-x-\frac{1}{\eta} \ln (-p)$, see Proposition 4.1, lead to

$$
\begin{aligned}
Y_{T}^{\epsilon}+\ell^{\epsilon}\left(X_{T}^{\epsilon}\right)-g\left(S_{T}\right)+\frac{1}{\eta} \ln \left(-P_{T}^{\epsilon}\right) \geq & Y_{T}^{\epsilon}-\psi^{\epsilon}\left(T, S_{T}, P_{T}^{\epsilon}, X_{T}^{\epsilon}\right)+\psi^{\epsilon}\left(T, S_{T}, P_{T}^{\epsilon}, X_{T}^{\epsilon}\right) \\
& +\ell^{\epsilon}\left(X_{T}^{\epsilon}\right)-g\left(S_{T}\right)+\frac{1}{\eta} \ln \left(-P_{T}^{\epsilon}\right) \\
\geq & Y_{T}^{\epsilon}-\psi^{\epsilon}\left(T, S_{T}, P_{T}^{\epsilon}, X_{T}^{\epsilon}\right)+\epsilon^{4} \check{\varpi} \circ \xi_{\epsilon}\left(T, S_{T}, X_{T}^{\epsilon}\right) \\
& -\epsilon^{3}\left|X_{T}^{\epsilon}\right| .
\end{aligned}
$$

Recall that $\check{\varpi} \geq 0$. We also know from (7.5) and (7.6) that $\left|X_{T}^{\epsilon}\right| \leq C_{K}$. Hence, we deduce from the above that

$$
Y_{T}^{\epsilon}+\ell^{\epsilon}\left(X_{T}^{\epsilon}\right)-g\left(S_{T}\right)+\frac{1}{\eta} \ln \left(-P_{T}^{\epsilon}\right) \geq Y_{T}^{\epsilon}-\psi^{\epsilon}\left(T, S_{T}, P_{T}^{\epsilon}, X_{T}^{\epsilon}\right)-C_{K} \epsilon^{3} .
$$

We now consider the right-hand side term in (7.8). Since $\pi_{p}>0$ and $\check{\varpi}$ do not depend on $p$, one can apply the expansion of Lemma 5.4 It implies

$$
E_{\epsilon}=\int_{t_{o}}^{T}\left(\frac{\sigma^{2}}{2} \eta \xi_{\epsilon}\left(\tau, S_{\tau}, X_{\tau}^{\epsilon}\right)^{2}+\frac{\sigma^{2}}{2} \delta^{2}\left(\check{\varpi}_{\xi \xi} \circ \xi_{\epsilon}\right)\left(\tau, S_{\tau}, X_{\tau}^{\epsilon}\right)+R_{\epsilon}\left(\tau, S_{\tau}, P_{\tau}^{\epsilon}, X_{\tau}^{\epsilon}\right)\right) d \tau
$$

in which the map $R_{\epsilon}$ is given by (5.13) for $\phi:=0$ and $w:=\check{\varpi}$.

Direct computations based on condition b. of Assumption 4.2, the specific forms of $\hat{a}$ and $\pi$, and (7.5) lead to $\left|R_{\epsilon}\right| \leq C_{K}$ on the closure of $\mathcal{J}_{\epsilon}$, and therefore: $\left|R_{\epsilon}\left(\cdot, S, P^{\epsilon}, X^{\epsilon}\right)\right| \leq$ $C_{K}$. It also follows from Assumption 4.2, (4.4) and (7.3) that $\left|\xi_{\epsilon}\left(\cdot, S, X^{\epsilon}\right)\right| \leq C_{K}$. Finally (6.5) above for the coefficients entering in the definition of $\check{\varpi}$ provides a uniform bound for the remaining term. Therefore

$$
\left|E_{\epsilon}\right| \leq C_{K} .
$$

Combining (7.8), (7.9) and (7.11) leads to

$$
Y_{T}^{\epsilon}+\ell^{\epsilon}\left(X_{T}^{\epsilon}\right)-g\left(S_{T}\right) \geq c-\frac{1}{\eta} \ln \left(-P_{T}^{\epsilon}\right)-C_{K} \epsilon^{2} .
$$

Recall that $C_{K}$ depends only on $K$ but not on $c$. Hence, we can choose $c=\left(C_{K}+1\right) \epsilon^{2}$, and obtain from the previous inequality that

$$
\Psi\left(Y_{T}^{\epsilon}+\ell^{\epsilon}\left(X_{T}^{\epsilon}\right)-g\left(S_{T}\right)\right) \geq P_{T}^{\epsilon} e^{-\eta \epsilon^{2}},
$$

so that

$$
\mathbb{E}\left[\Psi\left(Y_{T}^{\epsilon}+\ell^{\epsilon}\left(X_{T}^{\epsilon}\right)-g\left(S_{T}\right)\right)\right] \geq p_{o} e^{-\eta \epsilon^{2}},
$$

since $P^{\epsilon}$ is a martingale.

Step 3. Note that the strategy $L^{\epsilon}$ does not satisfy the admissibility condition (2.3). However, in Step 4, below we overcome this by replacing $L^{\epsilon}$ by an appropriately stopping it (see definition (7.15)). Towards this goal we start by proving below that the latter inequality implies that

$$
\sup _{L \in \mathfrak{L}^{\epsilon}\left(t_{o}, s_{o}, y_{o}, x_{o}\right)} \mathbb{E}\left[\Psi\left(\Delta^{\epsilon, L}\right)\right]>p_{o},
$$

in which we abbreviate notations by setting

$$
\Delta^{\epsilon, L}:=Y_{T}^{t_{o}, y_{o}, \epsilon, L}+\ell^{\epsilon}\left(X_{T}^{t_{o}, x_{o}, s_{o}, L}\right)-g\left(S_{T}^{t_{o}, s_{o}}\right) .
$$


Hence,

$$
\begin{aligned}
y_{o} & =v\left(t_{o}, s_{o}, p_{o}\right)+\epsilon^{4} \check{\varpi} \circ \xi_{\epsilon}\left(t_{o}, s_{o}, p_{o}, x_{o}\right)+\left(C_{K}+1\right) \epsilon^{2} \\
& \geq v^{\epsilon}\left(t_{o}, s_{o}, p_{o}, x_{o}\right),
\end{aligned}
$$

and therefore

$$
\begin{aligned}
u^{\epsilon}\left(t_{o}, s_{o}, p_{o}, x_{o}\right) & =\epsilon^{-2}\left(v^{\epsilon}-v\right)\left(t_{o}, s_{o}, p_{o}, x_{o}\right) \\
& \leq \epsilon^{2} \check{\varpi} \circ \xi_{\epsilon}\left(t_{o}, s_{o}, p_{o}, x_{o}\right)+\left(C_{K}+1\right)
\end{aligned}
$$

Recall that Assumption 4.2 implies that $\epsilon \check{\varpi} \circ \xi_{\epsilon}$ has linear growth in $x$, uniformly in its other variables and in $0<\epsilon \leq 1$, see Remark 3.5. The latter leads to the right-hand side inequality of (7.1).

Step 4. We now prove our claim (7.14). Recalling (7.5) and the fact that $g$ is bounded, (7.12) implies that

$$
Y_{T}^{\epsilon}+\ell^{\epsilon}\left(X_{T}^{\epsilon}\right) \geq-C_{K}-\int_{t_{o}}^{T} \gamma_{\tau}^{\epsilon} d W_{\tau},
$$

for some predictable process $\gamma^{\epsilon}$ which satisfies $\left|\gamma^{\epsilon}\right| \leq C_{K}$ for all $0<\epsilon \leq 1$. Then, it follows from 36 that

$$
\begin{aligned}
Y^{\epsilon}+\ell^{\epsilon}\left(X^{\epsilon}\right) & \geq-C_{K}-\mathbb{E}^{\mathbb{Q}}\left[\int_{t_{o}}^{T} \gamma_{\tau}^{\epsilon} d W_{\tau} \mid \mathcal{F} .\right] \\
& \geq-C_{K}+M^{\epsilon}
\end{aligned}
$$

in which $M^{\epsilon}:=-\int_{t_{o}}^{.} \gamma_{\tau}^{\epsilon} d W_{\tau}$ satisfies $\mathbb{E}\left[e^{2 \eta \sup _{\left[t_{o}, T\right]}\left|M^{\epsilon}\right|}\right] \leq C_{K}$.

Given $k \geq C_{K}$, we now denote by $\tau_{k}$ the first time after $t_{o}$ such that $Y^{\epsilon}+\ell^{\epsilon}\left(X^{\epsilon}\right)=$ $-k$. Set

$$
L^{\epsilon, k}:=L_{\cdot \wedge \tau_{k}}^{\epsilon} .
$$

Then, $L^{\epsilon}$ being continuous, $L^{\epsilon, k} \in \mathfrak{L}^{\epsilon}\left(t_{o}, s_{o}, y_{o}, x_{o}\right)$ for all $k \geq 1$. Moreover, since $\Psi \leq 0$,

$$
\Psi\left(\Delta^{\epsilon, L^{\epsilon}}\right)-\Psi\left(\Delta^{\epsilon, L^{\epsilon, k}}\right) \leq-\Psi(-k) \mathbf{1}_{\left\{\tau_{k} \leq T\right\}} \leq-\Psi(-k) \mathbf{1}_{\left\{\sup _{\left[t_{o}, T\right]}\left|M^{\epsilon}\right| \geq k-C_{K}\right\}} .
$$

We next use (7.13) and the Markov's inequality to obtain

$p_{o} e^{-\eta \epsilon^{2}} \leq \mathbb{E}\left[\Psi\left(\Delta^{\epsilon, L^{\epsilon}}\right)\right] \leq \mathbb{E}\left[\Psi\left(\Delta^{\epsilon, L^{\epsilon, k}}\right)\right]-\Psi(-k) C_{K} / e^{2 \eta k}=\mathbb{E}\left[\Psi\left(\Delta^{\epsilon, L^{\epsilon, k}}\right)\right]+C_{K} e^{-\eta k}$.

Then, taking

$$
k:=-\eta^{-1} \ln \left(p_{o}\left(e^{-\eta \epsilon^{2}}-1\right) / C_{K}\right)+1
$$

leads to (7.14), recall that $p_{o}<0$.

Step 5. It remains to explain how to consider the general case $\left(t_{o}, s_{o}, x_{o}\right) \in[0, T] \times$ $(0, \infty) \times \mathbb{R}$. First note that an immediate transfer allows one to pass from the initial position $\left(y_{o}, x_{o}\right)$ to $\left(y_{o}^{\prime}, x_{o}^{\prime}\right)$ with

$$
\begin{aligned}
y_{o}^{\prime} & :=y_{o}+\ell^{\epsilon}\left(x_{o}-x_{o}^{\prime}\right), \\
x_{o}^{\prime} & :=x_{o}+\left[-\epsilon \check{\xi}\left(t_{o}, s_{o}\right)+\theta\left(t_{o}, s_{o}\right)-x_{o}\right]^{+}-\left[x_{o}-\epsilon \check{\xi}\left(t_{o}, s_{o}\right)-\theta\left(t_{o}, s_{o}\right)\right]^{+} .
\end{aligned}
$$

By Remark [5.1. one has

$$
\begin{aligned}
v^{\epsilon}\left(t_{o}, s_{o}, p_{o}, x_{o}\right) & \leq v^{\epsilon}\left(t_{o}, s_{o}, p_{o}, x_{o}^{\prime}\right)+x_{o}^{\prime}-x_{o}+\epsilon^{3}\left|x_{o}-x_{o}^{\prime}\right| \\
& \leq v^{\epsilon}\left(t_{o}, s_{o}, p_{o}, x_{o}^{\prime}\right)+x_{o}^{\prime}-x_{o}+\epsilon^{3}\left(C_{K}+\left|x_{o}\right|\right)
\end{aligned}
$$


in which the last inequality follows from Assumption 4.2 Hence,

$$
\begin{aligned}
\left(v^{\epsilon}-v\right)\left(t_{o}, s_{o}, p_{o}, x_{o}\right) & \leq\left(v^{\epsilon}-v\right)\left(t_{o}, s_{o}, p_{o}, x_{o}^{\prime}\right)+x_{o}-x_{o}^{\prime}+x_{o}^{\prime}-x_{o}+\epsilon^{3}\left(C_{K}+\left|x_{o}\right|\right) \\
& \leq\left(v^{\epsilon}-v\right)\left(t_{o}, s_{o}, p_{o}, x_{o}^{\prime}\right)+\epsilon^{3}\left(C_{K}+\left|x_{o}\right|\right) .
\end{aligned}
$$

Since $\left(t_{o}, s_{o}, x_{o}^{\prime}\right)$ belongs to the closure of $\mathcal{J}_{\epsilon}$, we can apply the analysis of the preceding steps to conclude.

A by-product of the above argument is the explicit construction of a strategy $L^{\epsilon}$ which is $O\left(\epsilon^{2}\right)$-optimal for the problem with transaction costs. The constant $C_{K}$ in the following proposition can be recovered in terms of the constant $K$ of Assumption 4.2 .

Proposition 7.1. Let the conditions of Proposition 4.3 hold. Then, there exists a constant $C_{K}>0$ such that the following holds: Fix $\left(t_{o}, s_{o}, x_{o}, p_{o}\right) \in[0, T] \times(0, \infty) \times$ $\mathbb{R} \times(-\infty, 0), \epsilon \in(0,1)$, let

$$
y_{o}:=\psi^{\epsilon}\left(t_{o}, s_{o}, p_{o}, x_{o}\right)+\epsilon^{2}\left(C_{K}+1\right),
$$

where $\psi^{\epsilon}$ is defined as in (7.2), $\left(y_{o}^{\prime}, x_{o}^{\prime}\right)$ be defined as in (7.17)-(7.18), $L^{\epsilon, k}$ be given by the solution of (7.6)-(7.15) -(7.16) for the initial condition $\left(t_{o}, s_{o}, x_{o}^{\prime}, y_{o}^{\prime}\right)$, and $L^{\epsilon}:=$ $L^{\epsilon, k}+x_{o}^{\prime}-x_{o}$, then

$$
\mathbb{E}\left[\Psi\left(\Delta_{t_{o}, s_{o}, y_{o}, x_{o}}^{\epsilon, L^{\epsilon}}\right)\right] \geq p_{o} \text { and } y_{o}=v^{\epsilon}\left(t_{o}, s_{o}, p_{o}, x_{o}\right)+O\left(\epsilon^{2}\right) .
$$

Proof. We first prove the left-hand side inequality of (7.19). When $\left(t_{o}, s_{o}, x_{o}\right)$ belongs to the closure of $\mathcal{J}_{\epsilon}$ defined in (7.3), then $\left(x_{o}^{\prime}, y_{o}^{\prime}\right)=\left(x_{o}, y_{o}\right)$ and this is an immediate by-product of the construction made in the proof of Proposition 4.3. The general case is treated as in Step 5 of the proof of Proposition 4.3 , observing that

$\psi^{\epsilon}\left(t_{o}, s_{o}, p_{o}, x_{o}\right)=\psi^{\epsilon}\left(t_{o}, s_{o}, p_{o}, x_{o}^{\prime}\right)+x_{o}^{\prime}-x_{o}+\epsilon^{3}\left|x_{o}-x_{o}^{\prime}\right|=\psi^{\epsilon}\left(t_{o}, s_{o}, p_{o}, x_{o}^{\prime}\right)-\ell^{\epsilon}\left(x_{o}-x_{o}^{\prime}\right)$,

by Proposition 4.1 and (6.5).

To prove the right-hand side identity in (7.19), it suffices to use Proposition 4.3 and to recall (6.5):

$$
\left(\psi^{\epsilon}-v^{\epsilon}\right)\left(t_{o}, s_{o}, p_{o}, x_{o}\right)=\left(\psi^{\epsilon}-v\right)\left(t_{o}, s_{o}, p_{o}, x_{o}\right)+\left(v-v^{\epsilon}\right)\left(t_{o}, s_{o}, p_{o}, x_{o}\right)=O\left(\epsilon^{2}\right) .
$$

Under an additional regularity conditions, one can obtain a strategy which is optimal at the leading order $\epsilon^{2}$.

Proposition 7.2. Let the conditions of Proposition 4.3 hold. Assume further that $\left|s^{2} \delta_{s s}\right| \leq K$ on $\mathfrak{D}$. Then, there exists $C_{K}>0$ such that the following holds: Fix $\left(t_{o}, s_{o}, x_{o}, p_{o}\right) \in[0, T] \times(0, \infty) \times \mathbb{R} \times(-\infty, 0), \epsilon \in(0,1)$, set

$$
y_{o}:=\left(v+\epsilon^{2} \hat{u}+\epsilon^{4} \varpi \circ \xi_{\epsilon}\right)\left(t_{o}, s_{o}, p_{o}, x_{o}\right)+\epsilon^{3}\left(C_{K}+1\right),
$$

let $\left(y_{o}^{\prime}, x_{o}^{\prime}\right)$ be defined as in (7.17)-(7.18) with $\hat{\xi}$ in place of $\check{\xi}, L^{\epsilon, k}$ be given by the solution of (7.6)-(7.15) for $\mathcal{J}_{\epsilon}$ defined with $\hat{\xi}$ in place of $\dot{\xi}$ and for

$$
k:=-\eta^{-1} \ln \left(p_{o}\left(e^{-\eta \epsilon^{3}}-1\right) / C_{K}\right)+1
$$

and the initial condition $\left(t_{o}, s_{o}, x_{o}^{\prime}, y_{o}^{\prime}\right)$, and set $L^{\epsilon}:=L^{\epsilon, k}+x_{o}^{\prime}-x_{o}$, then

$$
\mathbb{E}\left[\Psi\left(\Delta_{t_{o}, s_{o}, y_{o}, x_{o}}^{\epsilon, L^{\epsilon}}\right)\right] \geq p_{o} \text { and } y_{o}=v^{\epsilon}\left(t_{o}, s_{o}, p_{o}, x_{o}\right)+O\left(\epsilon^{3}\right) .
$$


Proof. We only sketch the proof since it is a straightforward adaptation of the proof of Proposition 7.1, see also the proof of Proposition 4.5 below.

We follow line by line the arguments of the proof of Proposition 7.1 but with $\psi^{\epsilon}$ and $\mathcal{J}_{\epsilon}$ defined by

$$
\begin{aligned}
\psi^{\epsilon} & :=v+\epsilon^{2} \hat{u}+\epsilon^{4} \varpi \circ \xi_{\epsilon}, \\
\mathcal{J}_{\epsilon} & :=\left\{(t, s, x) \in[0, T] \times(0, \infty) \times \mathbb{R}:-\hat{\xi}(t, s)<\xi_{\epsilon}(t, s, x)<\hat{\xi}(t, s)\right\} .
\end{aligned}
$$

The fact that $\hat{u}$ is a classical solution of (3.5) while $\varpi$ solves (3.7) implies that the counterpart of (7.10) is

$$
E_{\epsilon}=\int_{t_{o}}^{T} R_{\epsilon}\left(\tau, S_{\tau}, P_{\tau}^{\epsilon}, X_{\tau}^{\epsilon}\right) d \tau
$$

where $R_{\epsilon}$ is given by (5.13) for $\phi:=\hat{u}$ and $w:=\varpi$. Observe that (7.5) remains in force since neither $\hat{u}$ nor $\varpi$ depend on $p$ and $s \hat{u}_{s}$ and $s \varpi_{s} \circ \xi_{\epsilon} \mathbf{1}_{\overline{\mathcal{J}}_{\epsilon}}$ are bounded. Under our additional assumptions, it is easy to check from the proof of Lemma 5.4, see (5.13), that $\left|E_{\epsilon}\right| \leq \epsilon C_{K}$ : the additional assumption that $s^{2} \delta_{s s}$ is bounded allows to control the term $\mathcal{L}_{S} \varpi$ in $R_{2}^{\epsilon}$ whereas the other terms are bounded by Assumption 4.2

\subsection{Power case}

We now provide the proof of Proposition 4.5. Since it is very close to the one of Proposition 4.3, we focus on the differences.

Proof of Proposition 4.5. We only show that, for any compact subset $B_{o} \subset$ $(-\infty, 0)$, there exists $c_{o}, \epsilon_{o}>0$ such that

$$
u^{\epsilon}(\zeta, x) \leq c_{o}(1+\epsilon|x|) \quad \text { for all }(\zeta, x) \in[0, T] \times(0, \infty) \times B_{o} \times \mathbb{R}, \epsilon \in\left(0, \epsilon_{o}\right] .
$$

From now on, we fix a compact subset $B_{o} \subset(-\infty, 0)$. We also fix another compact set $B \subset(-\infty, 0)$ such that $B_{o} \subset \operatorname{Int}(B)$, and denote by $C_{B}>0$ a generic constant that depends at most on $B$, and that may change from line to line. It will be clear later on that $B$ can be chosen in terms of $B_{o}$.

Step 1. We first deduce from (4.9) that, for $(t, p) \in[0, T] \times(-\infty, 0)$ one has

$$
\left\{\begin{array}{c}
\theta(t, p)=\frac{\lambda m(t)}{\sigma(1+\beta)}(-p)^{-\frac{1}{\beta}}, \delta(t, p)=\theta(t, p)\left(\frac{\lambda}{\sigma(1+\beta)}-1\right), \hat{a}(p)=\frac{\lambda \beta}{\beta+1}(-p), \\
h(t, p)=\frac{\sigma^{2}}{2} \frac{\pi_{p p}}{\left(\pi_{p}\right)^{2}}(t, p) \hat{\xi}(t, p)^{2}, \hat{\xi}(t, p)=\left(\frac{3}{2} \delta(t, p)^{2} \frac{\left(\pi_{p}\right)^{2}}{\pi_{p p}}(t, p)\right)^{\frac{1}{3}} .
\end{array}\right.
$$

Let $(\varpi, h)$ be defined as in Lemma 3.4 and note that $(\varpi(\cdot, \xi), h)$ depends only on $p$, for $\xi \in \mathbb{R}$. Let $\hat{u}$ be the solution of (3.7). It is not difficult to deduce from (7.21) that one has

$$
f(t, p)=f(t,-1)(-p)^{-\frac{1}{\beta}} \quad \text { with } f(\cdot,-1) \in C_{b}^{\infty}([0, T]), \quad f \in\{\theta, \delta, \hat{\xi}, h, \hat{u}\} .
$$

We set

$$
\psi^{\epsilon}=v+\epsilon^{2} \hat{u}+\epsilon^{4} \varpi \circ \xi_{\epsilon} \quad, \quad \hat{a}^{\epsilon}:=\frac{-\bar{\sigma}_{0}^{\top} D \psi^{\epsilon}}{\psi_{p}^{\epsilon}}
$$

and

$$
\mathcal{J}_{\epsilon}:=\left\{(t, p, x) \in[0, T] \times(-\infty, 0) \times \mathbb{R}:-\hat{\xi}(t, p)<\xi_{\epsilon}(t, p, x)<\hat{\xi}(t, p)\right\} .
$$


We now fix $\left(t_{o}, s_{o}, x_{o}\right)$ in the closure of $\mathcal{J}_{\epsilon}$, the general case being handled as in Step 5 of the proof of Proposition 4.3. We let $p_{o} \in B$ and

$$
y_{o}:=c+\psi^{\epsilon}\left(t_{o}, s_{o}, p_{o}, x_{o}\right)
$$

for some $c>0$ to be chosen later on. We next define $\left(Y^{\epsilon}, X^{\epsilon}, S, L^{\epsilon}, P^{\epsilon}\right)$ as in the proof of Proposition 4.3 but with $(\varpi, \hat{\xi})$ in place of $(\check{\varpi}, \check{\xi})$, namely

$$
\left\{\begin{array}{l}
P^{\epsilon}=p_{o}+\int_{t_{o}} \hat{a}^{\epsilon}\left(\tau, S_{\tau}, P_{\tau}^{\epsilon}, X_{\tau}^{\epsilon}\right) d W_{\tau}, \\
X^{\epsilon}=x_{o}+\int_{t_{o}} X_{\tau}^{\epsilon} \frac{d S_{\tau}}{S_{\tau}}+\int_{t_{o}} d L_{\tau}^{\epsilon+}-\int_{t_{o}} d L_{\tau}^{\epsilon-}, \\
\left(\cdot, P^{\epsilon}, X^{\epsilon}\right) \in \mathcal{J}_{\epsilon} d t \otimes d \mathbb{P} \text {-a.e. on }\left[t_{o}, T\right], \\
L^{\epsilon \pm}=\int_{t_{o}} \chi_{\left\{\left(\tau, P_{\tau}^{\epsilon}, X_{\tau}^{\epsilon}\right) \in \partial \mathcal{J}_{\epsilon}^{ \pm}\right\}} d L_{\tau}^{\epsilon \pm},
\end{array}\right.
$$

and

$$
Y^{\epsilon}=y_{o}-\int_{t_{o}}^{\cdot}\left(1+\epsilon^{3}\right) d L_{\tau}^{\epsilon+}+\int_{t}^{\cdot}\left(1-\epsilon^{3}\right) d L_{\tau}^{\epsilon-}, y_{o}:=c+\psi^{\epsilon}\left(t_{o}, s_{o}, p_{o}, x_{o}\right) .
$$

We claim that a solution exists and that, for all $q>0$, there exists $C_{B}^{q}>0$, which depends only on $B$ and $q$, such that

$$
\sup _{\epsilon \in(0,1]} \mathbb{E}\left[\sup _{t \in\left[t_{0}, T\right]}\left(\left|P_{t}^{\epsilon}\right|^{q}+\left|P_{t}^{\epsilon}\right|^{-q}\right)\right] \leq C_{B}^{q} .
$$

This will be proved in Step 3 below. Since $\hat{u}, \varpi \geq 0$, and $\hat{u}$ does not depend on $x$, the same arguments as in Step 2 of the proof of Proposition 4.3 leads to

$$
Y^{\epsilon}+\ell^{\epsilon}\left(X^{\epsilon}\right) \geq c+\psi^{\epsilon}\left(\cdot, S, P^{\epsilon}, 0\right)-\epsilon^{2} E_{\epsilon}(\cdot) \geq c+v\left(\cdot, S, P^{\epsilon}, 0\right)-\epsilon^{2} E_{\epsilon},
$$

where

$$
\begin{aligned}
E_{\epsilon} & :=\epsilon\left|X^{\epsilon}\right|+\int_{t_{o}}\left(\frac{\sigma^{2}}{2} \frac{\pi_{p p}}{\left(\pi_{p}\right)^{2}} \xi_{\epsilon}^{2}+\frac{\sigma^{2}}{2} \delta^{2}\left(\varpi_{\xi \xi} \circ \xi_{\epsilon}\right)+\mathcal{H} \hat{u}+R_{\epsilon}\right)\left(\tau, X_{\tau}^{\epsilon}, P_{\tau}^{\epsilon}\right) d \tau \\
& =\epsilon\left|X^{\epsilon}\right|+\int_{t_{o}} R_{\epsilon}\left(\tau, S_{\tau}, P_{\tau}^{\epsilon}, X_{\tau}^{\epsilon}\right) d \tau
\end{aligned}
$$

in which the second equality follows from the fact that $\hat{u}$ and $\varpi$ solve (3.5) and (3.7) respectively, and $R_{\epsilon}$ is defined in (5.13) for $\phi:=\hat{u}$ and $w:=\varpi$. Observe that all the functions in the definition of $R_{\epsilon}$ are powers of the $p$-variable multiplied, at least, by $\epsilon$. Moreover, the definition of $X^{\epsilon}$ combined with (7.23) and (7.21) implies that $X^{\epsilon}$ is also controlled by a polynomial in $\left|P^{\epsilon}\right|$. Namely, we can find $q_{\beta}, C_{\beta}>0$, which only depend on $\beta$, such that

$$
\epsilon^{-1} \int_{t_{o}}^{t}\left|R_{\epsilon}\left(\tau, S_{\tau}, P_{\tau}^{\epsilon}, X_{\tau}^{\epsilon}\right)\right| d \tau+\left|X_{t}^{\epsilon}\right| \leq \Gamma_{t}^{\epsilon}:=C_{\beta} \sup _{\left[t_{o}, t\right]}\left(1+\left|P^{\epsilon}\right|^{-q_{\beta}}+\left|P^{\epsilon}\right|^{q_{\beta}}\right), \quad t \in\left[t_{o}, T\right] .
$$

We now take $c=3 \epsilon^{5 / 2}$. Since $v \geq-\kappa$, (7.27) implies

$$
Y^{\epsilon}+\ell^{\epsilon}\left(X^{\epsilon}\right) \geq-\kappa+2 \epsilon^{5 / 2}+\epsilon^{5 / 2}\left(1-\epsilon^{1 / 2} \Gamma^{\epsilon}\right) .
$$

Let $\tau_{\epsilon}$ be the first time such that $Y^{\epsilon}+\ell^{\epsilon}\left(X^{\epsilon}\right)$ is equal to $\epsilon^{5 / 2}-\kappa$. We let $\left(\tilde{Y}^{\epsilon}, \tilde{X}^{\epsilon}\right)$ be defined by the strategy in which we follow $L^{\epsilon}$ on $\left[t_{o}, \tau_{\epsilon}\left[\right.\right.$ and liquidate the position at $\tau_{\epsilon}$, i.e.

$$
\left(\tilde{Y}^{\epsilon}, \tilde{X}^{\epsilon}\right)=\left(Y^{\epsilon}, X^{\epsilon}\right) \mathbf{1}_{\llbracket t_{o}, \tau_{\epsilon} \wedge T \llbracket}+\left(Y_{\tau_{\epsilon} \wedge T}^{\epsilon}, \ell^{\epsilon}\left(X_{\tau_{\epsilon} \wedge T}^{\epsilon}\right)\right) \mathbf{1}_{\llbracket \tau_{\epsilon} \wedge T, T \rrbracket} .
$$


Note that this strategy is admissible by construction. Set $A_{\epsilon}:=\left\{\epsilon^{\frac{1}{2}} \Gamma_{T}^{\epsilon} \leq 1\right\}$. The inclusion $A_{\epsilon} \subset\left\{\tau_{\epsilon} \geq T\right\}$ follows from the last inequality and the fact that $\Gamma^{\epsilon}$ is nondecreasing. We then obtain

$$
\begin{aligned}
\mathbb{E}\left[\Psi\left(\tilde{Y}_{T}^{\epsilon}+\ell^{\epsilon}\left(\tilde{X}_{T}^{\epsilon}\right)\right)\right] & \geq \mathbb{E}\left[\Psi\left(2 \epsilon^{5 / 2}+\Phi\left(P_{T}^{\epsilon}\right)\right) \mathbf{1}_{A_{\epsilon}}\right]-\left|\Psi\left(\epsilon^{5 / 2}-\kappa\right)\right| \mathbb{P}\left[A_{\epsilon}^{c}\right] \\
& \geq \mathbb{E}\left[P_{T}^{\epsilon}\right]-\left(\mathbb{E}\left[\left|P_{T}^{\epsilon}\right|^{2}\right]^{\frac{1}{2}}+\left|\Psi\left(\epsilon^{5 / 2}-\kappa\right)\right|\right) \mathbb{P}\left[A_{\epsilon}^{c}\right]^{\frac{1}{2}} \\
& =p_{o}-\left(\mathbb{E}\left[\left|P_{T}^{\epsilon}\right|^{2}\right]^{\frac{1}{2}}+\left|\Psi\left(\epsilon^{5 / 2}-\kappa\right)\right|\right) \mathbb{P}\left[A_{\epsilon}^{c}\right]^{\frac{1}{2}}
\end{aligned}
$$

in which we used the fact that $P^{\epsilon}$ is a martingale by (7.26). We now appeal to (7.26) and (7.28) to obtain

$\mathbb{E}\left[\left|P_{T}^{\epsilon}\right|^{2}\right] \leq C_{B} \quad, \quad\left|\Psi\left(\epsilon^{5 / 2}-\kappa\right)\right|=\frac{1}{\epsilon^{5 \beta / 2}}$ and $\mathbb{P}\left[A_{\epsilon}^{c}\right] \leq \epsilon^{6+5 \beta} \mathbb{E}\left[\left|\Gamma_{T}^{\epsilon}\right|^{12+10 \beta}\right] \leq \epsilon^{5 \beta} C_{B} \epsilon^{6}$

Combining the above shows that, for some $c_{B}>0$, which only depends on $B$,

$$
\mathbb{E}\left[\Psi\left(\tilde{Y}_{T}^{\epsilon}+\ell^{\epsilon}\left(\tilde{X}_{T}^{\epsilon}\right)\right)\right] \geq p_{o}-c_{B} \epsilon^{3}
$$

and therefore, by (7.24), our choice $c=3 \epsilon^{5 / 2}$, the fact that $\hat{u}, \varpi$ satisfy (7.22) and that $v^{\epsilon}$ is non-decreasing in $p$,

$$
v^{\epsilon}\left(t_{o}, s_{o}, p_{o}-\tilde{c}_{B} \epsilon^{3}, x_{o}\right) \leq v\left(t_{o}, s_{o}, p_{o}, x_{o}\right)+\tilde{c}_{B} \epsilon^{5 / 2},
$$

for some constant $\tilde{c}_{B}>0$ that only depends on $B$.

Step 2. Since $\tilde{c}_{B}$ does not depend on $p_{o} \in B$, the above is true for any $p \in B$ in place of $p_{o}$. Set $\iota(p):=p+\epsilon^{5 / 2}$ for $p \in B_{o}$, recall that $B_{o} \subset \operatorname{Int}(B)$. Then,

$$
0>\iota(p)-\tilde{c}_{B} \epsilon^{3}=p+\epsilon^{5 / 2}-\tilde{c}_{B} \epsilon^{3} \geq p \text { for all } p \in B_{o} \text { and } 0<\epsilon \leq \epsilon_{B},
$$

for some $\epsilon_{B} \in(0,1)$ such that $p+\epsilon_{B}^{5 / 2} \in B$ for all $p \in B_{o}$. For the rest of the proof, we assume that $p_{o} \in B_{o}$. Then, (7.30) applied to $\iota\left(p_{o}\right)$ in place of $p_{o}$ and the fact that $v^{\epsilon}$ is non-decreasing in $p$ imply that

$$
v^{\epsilon}\left(t_{o}, s_{o}, p_{o}, x_{o}\right) \leq v^{\epsilon}\left(t_{o}, s_{o}, \iota\left(p_{o}\right)-\tilde{c}_{B} \epsilon^{3}, x_{o}\right) \leq v\left(t_{o}, s_{o}, \iota\left(p_{o}\right), x_{o}\right)+\tilde{c}_{B} \epsilon^{5 / 2} .
$$

We now use (4.9) to obtain

$$
v^{\epsilon}\left(t_{o}, s_{o}, p_{o}, x_{o}\right) \leq v\left(t_{o}, s_{o}, p_{o}, x_{o}\right)+\epsilon^{5 / 2} \beta^{-1}\left|m\left(t_{o}\right)\right|\left|p_{o}+\epsilon_{B}^{5 / 2}\right|^{-\frac{1}{\beta}-1}+\tilde{c}_{B} \epsilon^{5 / 2} .
$$

This proves (7.20).

Step 3. It remains to prove our claim. Using (7.22) and 6.5) below, we obtain that $\hat{a}^{\epsilon}$ is locally Lispchitz on $\mathcal{J}_{\epsilon}$ and that there exists a function $f \in C_{b}^{\infty}([0, T])$ such that

$$
\left|\hat{a}^{\epsilon}(t, p, x)\right| \leq \frac{(-p)^{\frac{1}{\beta}+1}}{m(t)+\epsilon^{2} f(t)}\left|-\sigma x+\epsilon^{4} \sigma x \varpi_{\xi} \circ \xi_{\epsilon}(t, p, x)\right|,(t, p, x) \in[0, T] \times(-\infty) \times \mathbb{R} .
$$

It follows from (7.22) and (ii) of Lemma 3.4 that

$$
\left|\hat{a}^{\epsilon}(t, p, x)\right| \leq C_{K}|p| \quad \text { for }(t, p, x) \in \mathcal{J}_{\epsilon},
$$

and for $\epsilon$ small enough with respect to $f$ and $m$. In particular, the existence to the system (7.25) will automatically imply (7.26). For $\rho>0$, set $B_{\rho}:=\left[-e^{\rho},-e^{-\rho}\right]$ and let $\hat{a}^{\epsilon, \rho}$ be a Lipschitz function such that $\hat{a}^{\epsilon, \rho}=\hat{a}^{\epsilon}$ on $[0, T+1] \times B_{\rho} \times \mathbb{R}$ and $\hat{a}^{\epsilon, \rho}=0$ on $[0, T+1] \times B_{2 \rho}^{c} \times \mathbb{R}$. Here all functions are extended to $[0, T+1]$ by taking their 
values at $T$ on $[T, T+1]$. The set $\mathcal{J}_{\epsilon}^{\rho}:=\left([0, T+1] \times\left(B_{2 \rho}\right)^{c} \times \mathbb{R}\right) \cap \mathcal{J}_{\epsilon}$ is bounded and it follows from [25] that there exists a strong solution $\left(P^{\epsilon, \rho}, X^{\epsilon, \rho}\right)$ to (7.25) with $\hat{a}^{\epsilon, \rho}$ in place of $\hat{a}^{\epsilon}$. Let $\tau_{\epsilon}^{\rho}$ be the first time after $t_{o}$ when $P^{\epsilon, \rho}$ reaches the boundary of $B_{\rho}$. For $\rho>\left|\ln \left(-p_{o}\right)\right|,\left(X^{\epsilon, \rho}, P^{\epsilon, \rho}\right)$ solves (7.25) on $\llbracket t_{o}, \tau_{\epsilon}^{\rho} \wedge T \rrbracket$. It follows from (7.31) that $\tau_{\epsilon}^{\rho} \wedge(T+1)$ converges to $T+1$ in probability as $\rho \rightarrow \infty$. Hence, after possibly passing to a subsequence $\left(\tau_{\epsilon}^{\rho_{n}}\right)_{n \geq 1}$, it converges almost surely to $T+1$ as $n \rightarrow \infty$. Let us set

$$
\left(X^{\epsilon}, P^{\epsilon}\right):=\left(x_{o}, p_{o}\right) \mathbf{1}_{\left\{t_{o}\right\}}+\sum_{n \geq 1} \mathbf{1}_{\rrbracket \tau_{\epsilon}^{\rho_{n-1}} \wedge T, \tau_{\epsilon}^{\rho_{n}} \wedge T \rrbracket}\left(X^{\epsilon, \rho_{n}}, P^{\epsilon, \rho_{n}}\right)
$$

with the convention $\tau_{\epsilon}^{\rho_{0}}:=t_{0}$. Since $\left(X^{\epsilon, \rho_{n}}, P^{\epsilon, \rho_{n}}\right)=\left(X^{\epsilon, \rho_{n+k}}, P^{\epsilon, \rho_{n+k}}\right)$ on $\llbracket t_{o}, \tau_{\epsilon}^{\rho_{n}} \wedge T \rrbracket$, for all $k \geq 1$, it solves (7.25) on each $\llbracket t_{o}, \tau_{\epsilon}^{\rho_{n}} \wedge T \rrbracket, n \geq 1$. Since $\left(\tau_{\epsilon}^{\rho_{n}} \wedge(T+1)\right)_{n \geq 1}$ converges almost surely to $T+1$ as $n \rightarrow \infty,\left(X^{\epsilon}, P^{\epsilon}\right)$ solves (7.25) on $\left[t_{o}, T\right]$.

Remark 7.3. The same arguments as in the proof of Propositions 7.1 and 7.2 show that the above allows to construct a strategy $L^{\epsilon}$, based on the sole knowledge of $v, \hat{u}$, $\varpi$ and $\theta$, satisfying $\mathbb{E}\left[\Psi\left(\Delta_{t_{o}, s_{o}, y_{o}, x_{o}}^{\epsilon, L^{\epsilon}}\right)\right] \geq p_{o}$ for

$$
\begin{aligned}
y_{o} & =\left(v+\epsilon^{2} \hat{u}+\epsilon^{4} \varpi \circ \xi_{\epsilon}\right)\left(t_{o}, s_{o}, p_{o}, x_{o}\right)+C \epsilon^{5 / 2} \\
& =v^{\epsilon}\left(t_{o}, s_{o}, p_{o}, x_{o}\right)+o\left(\epsilon^{2}\right),
\end{aligned}
$$

where $C>0$ can be computed explicitly.

\section{Appendix}

We provide here the proofs of Theorem 2.1. Theorem 2.3 and Proposition 2.2 for completeness. These results are essentially known but our framework requires some slight adjustments.

Proof of Theorems 2.1 and 2.3: We focus on the proof of Theorem 2.1. Theorem 2.3 is proved by combining the following arguments with the results of [12] instead of 9]. The arguments of [9] can not be applied per-se to obtain Theorem 2.1 because their Standing Assumption 4 may not hold in our context. We explain briefly how to modify it. First, this does not alter the proof of (GDP1) in [9, Corollary 2.9], which in turn leads to the viscosity supersolution property by the same arguments as in [9, Section 5]. Similarly, the proof of the subsolution property [9, Section 5] can be reproduced once (GDP2) stated in 9, Corollary 2.9] is valid. It is the case, by [9], if one imposes the additional constraints $Y^{t, y, \epsilon, L}+\ell^{\epsilon}\left(X^{t, x, s, L}\right) \geq-c$ on $[t, T]$, with $c>0$ fixed independent of the control $L$. Their standing Assumption 4 is then satisfied, see [36, Lemma 3.3] which imposes a uniform $L^{2}$ bound on the admissible controls $L$. Then, the corresponding value function $v^{\epsilon, c}$ satisfies that its upper-semicontinuous envelope $v^{\epsilon, c *}$ is a viscosity subsolution of (2.7) on $\left\{v^{\epsilon, c, *}(t, s, p, x)+\ell^{\epsilon}(x)>-c\right\}$, by [9, Section 5]. The sequence of corresponding operators converges to the one of (2.7) as $c \rightarrow \infty$. By standard stability results for viscosity solutions, see e.g. 4, this implies that the relaxed semi-limit $v^{\epsilon, \infty *}$ defined by $v^{\epsilon, \infty *}(t, s, p, x):=\lim \sup _{\left(c, t^{\prime}, s^{\prime}, p^{\prime}, x^{\prime}\right) \rightarrow(\infty, t, s, p, x)} v^{\epsilon, c *}\left(t^{\prime}, s^{\prime}, p^{\prime}, x^{\prime}\right)$ is a viscosity subsolution of (2.7). Note that $v^{\epsilon, \infty *} \geq v^{\epsilon *}$ by monotonicity. It remains to check that the converse inequality holds. But the admissibility constraint entering in the definition of $\mathfrak{L}^{\epsilon}$ means that, for all $\iota>0$, we can find $c_{\iota}>0$ such that $v^{\epsilon, c_{\iota}} \leq v^{\epsilon}+\iota \leq v^{\epsilon *}+\iota$.

Proof of Proposition 2.2 Let us first fix $z>\pi(t, s, p)$. Then, we can find $(\vartheta, \alpha) \in$ $\mathcal{U} \times \mathfrak{A}$ such that $\Psi\left(Z_{T}^{t, s, z, \vartheta}-g\left(S_{T}^{t, s}\right)\right) \geq P_{T}^{t, p, \alpha}$. Recall from the discussion after (2.6) that we can restrict $P_{T}^{t, p, \alpha}$ to take values in the image of $\Psi$, and therefore in the domain of 
definition of $\Phi$. Since $\Phi$ is non decreasing, it follows that $Z_{T}^{t, s, z, \vartheta} \geq g\left(S_{T}^{t, s}\right)+\Phi\left(P_{T}^{t, p, \alpha}\right)$. Then, the convexity of $\Phi$ and the fact that $\Phi^{\prime} \circ I$ is the identity imply

$$
\begin{aligned}
Z_{T}^{t, s, z, \vartheta} & \geq g\left(S_{T}^{t, s}\right)+\Phi \circ I\left(\hat{q} Q_{T}^{t, s}\right)+\Phi^{\prime} \circ I\left(\hat{q} Q_{T}^{t, s}\right)\left(P_{T}^{t, p, \alpha}-I\left(\hat{q} Q_{T}^{t, s}\right)\right) \\
& =g\left(S_{T}^{t, s}\right)+\Phi \circ I\left(\hat{q} Q_{T}^{t, s}\right)+\hat{q} Q_{T}^{t, s}\left(P_{T}^{t, p, \alpha}-I\left(\hat{q} Q_{T}^{t, s}\right)\right) .
\end{aligned}
$$

We conclude by taking expectation under $\mathbb{Q}^{t, s}$. Since $Z^{t, s, z, \vartheta}$ is a $\mathbb{Q}^{t, s}$-supermartingale, as a local-martingale bounded from below, and $P^{t, p, \alpha}$ a $\mathbb{P}$-martingale, the definition of $\hat{q}$ and $\mathbb{Q}^{t, s}$ lead to $y \geq \gamma+\hat{q}(p-p)=\gamma$, where $\gamma$ denotes the right-hand side term in (2.9). This shows that $\pi(t, s, p) \geq \gamma$.

To see that the reverse inequality holds, just observe that our integrability condition imply that we can find $\vartheta_{n} \in \mathcal{U}\left(t, s, z_{n}\right)$ such that

$$
Z_{T}^{t, s, z_{n}, \vartheta_{n}}=H_{n}:=\left(g\left(S_{T}^{t, s}\right)+\Phi \circ I\left(\hat{q} Q_{T}^{t, s}\right)\right) \vee(-n),
$$

in which $z_{n}:=\mathbb{E}^{\mathbb{Q}^{t, s}}\left[H_{n}\right] \downarrow \gamma$ as $n \rightarrow \infty$. Then, $\mathbb{E}\left[\Psi\left(H_{n}-g\left(S_{T}^{t, s}\right)\right)\right] \downarrow \mathbb{E}\left[I\left(\hat{q} Q_{T}^{t, s}\right)\right]=p$, by monotone convergence and definition of $\hat{q}$.

\section{References}

[1] A. Altarovici, J. Muhle-Karbe, and H.M. Soner. Asymptotics for fixed transaction costs. Preprint, arXiv:1306.2802, 2013.

[2] C. Atkinson and S. Mokkhavesa. Multi-asset portfolio optimization with transaction cost. Applied Mathematical Finance, 11(2):95-123, 2004.

[3] G. Barles and H.M. Soner. Option pricing with transaction costs and a nonlinear black-scholes equation. Finance and Stochastics, 2(4):369-397, 1998.

[4] G. Barles and P.E. Souganidis. Convergence of approximation schemes for fully nonlinear second order equations. Asymptotic Analysis, 4:271-283, 1991.

[5] M. Bichuch. Pricing a contingent claim liability with transaction costs using asymptotic analysis for optimal investment. Preprint, arXiv:1112.3012, 2011.

[6] M. Bichuch. Asymptotic analysis for optimal investment in finite time with transaction costs. SIAM Journal on Financial Mathematics, 3(1):433-458, 2012.

[7] M. Bichuch and S.E. Shreve. Utility maximization trading two futures with transaction costs. SIAM Journal on Financial Mathematics, 4(1):26-85, 2013.

[8] B. Bouchard. Utility maximization on the real line under proportional transaction costs. Finance Stoch., 6(4):495-516, 2002.

[9] B. Bouchard and N.M. Dang. Generalized stochastic target problems for pricing and partial hedging under loss constraints - application in optimal book liquidation. Finance and Stochastics, 17:1-42, 2010.

[10] B. Bouchard, R. Elie, and C. Imbert. Optimal control under stochastic target constraints. SIAM Journal on Control and Optimization, 48 (5):3501-3531, 2010.

[11] B. Bouchard, R. Elie, and A. Réveillac. Bsdes with weak terminal condition. To appear in The Annals of Probability.

[12] B. Bouchard, R. Elie, and N. Touzi. Stochastic target problems with controlled loss. SIAM J. Control Optim., 48(5):3123-3150, 2009/10. 
[13] B. Bouchard, L. Moreau, and M. Nutz. Stochastic target games with controlled loss. The Annals of Applied Probability, 24(3), 899-934, 2014.

[14] B. Bouchard and M. Nutz. Weak dynamic programming for generalized state constraints. SIAM Journal on Control and Optimization, 50(6):3344-3373, 2012.

[15] B. Bouchard and N. Touzi. Explicit solution to the multivariate super-replication problem under transaction costs. Annals of Applied Probability, 10:685-708, 2000.

[16] B. Bouchard and T.N. Vu. A stochastic target approach for p\&l matching problems. Mathematics of Operations Research, 37:526-558, 2012.

[17] G.M. Constantinides. Capital market equilibrium with transaction costs. The Journal of Political Economy, 94(4): 842-862, 1986.

[18] M.G. Crandall, H. Ishii, and P.L. Lions. User's guide to viscosity solutions of second order partial differential equations. Amer. Math. Soc., 27:1-67, 1992.

[19] J. Cvitanić, H. Pham, and N. Touzi. A closed-form solution to the problem of super-replication under transaction costs. Finance and Stochastics, 3(1):35-54, 1999.

[20] J. Cvitanić, H. Pham, and N. Touzi. Super-replication in stochastic volatility models under portfolio constraints. Journal of Applied Probability, 36(2):523-545, 1999.

[21] M.H. Davis, V.C. Panas, and T. Zariphopoulos. European option pricing with transaction costs. SIAM J. Control Optim., 31(2):470-493, 1993.

[22] M.H. Davis and A.R. Norman. Portfolio selection with transaction costs. Mathematics of Operations Research, 15(4):676-713, 1990.

[23] F. Delbaen, Y.M. Kabanov, and E. Valkeila. Hedging under transaction costs in currency markets: a discrete-time model. Mathematical Finance, 12(1):45-61, 2002.

[24] B. Dumas and E. Luciano. An exact solution to a dynamic portfolio choice problem under transactions costs. The Journal of Finance, 46(2):577-595, 1991.

[25] P. Dupuis and H. Ishii. SDEs with oblique reflection on nonsmooth domains. The Annals of Probability, 21(1):554-580, 1993.

[26] L.C. Evans. Periodic homogenisation of certain fully nonlinear partial differential equations. Proc. Roy. Soc. Edinburgh Sect. A, 120(3-4):245-265, 1992.

[27] H. Föllmer and P. Leukert. Quantile hedging. Finance and Stochastics, 3:251-273, 1999.

[28] H. Föllmer and P. Leukert. Efficient hedging: cost versus shortfall risk. Finance and Stochastics, 4:117-146, 2000.

[29] J.-P. Fouque, R. Sircar, and T. Zariphopoulou. Portfolio Optimization and Stochastic Volatility Asymptotics. Preprint 2013.

[30] J.-P. Fouque, G. Papanicolaou, and R. Sircar. Derivatives in Financial Markets with Stochastic Volatility. Cambridge University Press, 2000.

[31] J.-P. Fouque, G. Papanicolaou, R. Sircar, and K. Sølna. Multiscale Stochastic Volatility for Equity, Interest-Rate and Credit Derivatives. Cambridge University Press, 2011. 
[32] S. Gerhold, J. Muhle-Karbe, and W. Schachermayer. Asymptotics and duality for the Davis and Norman problem. Stochastics An International Journal of Probability and Stochastic Processes, 84(5-6):625-641, 2012.

[33] J. Goodman and D.N. Ostrov. Balancing small transaction costs with loss of optimal allocation in dynamic stock trading strategies. SIAM Journal on Applied Mathematics, 70(6):1977-1998, 2010.

[34] S.D. Hodges and A. Neuberger. Optimal replication of contingent claims under transaction costs. Review of futures markets, 8(2):222-239, 1989.

[35] K. Janecek and S.E. Shreve. Asymptotic analysis for optimal investment and consumption with transaction costs. Finance and Stochastics, 8(2):181-206, 2004.

[36] Y.M. Kabanov. Hedging and liquidation under transaction costs in currency markets. Finance and Stochastics, 3:237-248, 1999.

[37] Y.M. Kabanov and G. Last. Hedging under transaction costs in currency markets: a continuous-time model. Mathematical Finance, 12(1):63-70, 2002.

[38] Y.M. Kabanov and M.M. Safarian. Markets with transaction costs. Springer, 2009.

[39] I. Karatzas and S.E. Shreve. Brownian motion and stochastic calculus, volume 113. Springer Verlag, 1991.

[40] P.F. Koehl, H. Pham, and N. Touzi. Hedging in discrete time under transaction costs and continuous-time limit. Journal of applied probability, 36(1):163-178, 1999.

[41] P.F. Koehl, H. Pham, and N. Touzi. On super-replication under transaction costs in general discrete-time models. Theory of Probability and its Applications, 45:783788,1999 .

[42] S. Levental and A.V. Skorohod. On the possibility of hedging options in the presence of transaction costs. Ann. Appl. Probab., 7:410-443, 1997.

[43] M.J.P. Magill and G.M. Constantinides. Portfolio selection with transactions costs. Journal of Economic Theory, 13(2):245-263, 1976.

[44] L. Moreau. Stochastic target problems with controlled loss in jump diffusion models. SIAM J. Control Optim., 49(6):2577-2607, 2011.

[45] L. Moreau, J. Muhle-Karbe, and H.M. Soner. Portfolio choice with small market impact. Preprint, 2013.

[46] D. Possamai and G. Royer. General indifference pricing with small transaction costs. Preprint, 2013.

[47] D. Possamai, H.M. Soner, and N. Touzi. Homogenization and asymptotics for small transaction costs: the multidimensional case. Preprint, arXiv:1212.6275, 2012.

[48] D. Possamai, H.M. Soner, and N. Touzi. Large liquidity expansion of super-hedging costs. Asymptotic Analysis, 79(1):45-64, 2012.

[49] S.E. Shreve and H.M. Soner. Optimal investment and consumption with transaction costs. Ann. Appl. Probab., 4(3):609-692, 1994.

[50] H.M. Soner, S.E. Shreve, and J. Cvitanic. There is no nontrivial hedging portfolio for option pricing with transaction costs. Ann. Appl. Prob., 5:327-355, 1995. 
[51] H.M. Soner and N. Touzi. Dynamic programming for stochastic target problems and geometric flows. Journal of the European Mathematical Society, 4(3):201-236, 2002.

[52] H.M. Soner and N. Touzi. Stochastic target problems, dynamic programming, and viscosity solutions. SIAM Journal on Control and Optimization, 41(2):404-424, 2002.

[53] H.M. Soner and N. Touzi. Homogenization and asymptotics for small transaction costs. SIAM Journal on Control and Optimization, 51(4), 2893-2921, 2013.

[54] A.E. Whalley and P. Wilmott. An asymptotic analysis of an optimal hedging model for option pricing with transaction costs. Mathematical Finance, 7(3):307324, 1997. 